\title{
To Transmit Now or Not To Transmit Now
}

\author{
Dacfey Dzung ${ }^{\S}$, Rachid Guerraoui ${ }^{\dagger}$, David Kozhaya ${ }^{\dagger *}$ and Yvonne-Anne Pignolet ${ }^{\S}$ \\ ${ }^{\S}$ ABB Corporate Research, Switzerland \\ ${ }^{\dagger}$ EPFL, Switzerland \\ Email: firstname.lastname@ $\left\{{ }^{\S}\right.$ ch.abb.com; †epfl.ch $\}$
}

\begin{abstract}
This paper studies how a reliable communication service can be built, with high probability synchrony, over a wireless channel. We investigate environments where energy consumption is crucial, e.g., sensor networks. We consider a Partially Observable Markov Decision Process (POMDP) setting in which the channel state: (i) changes according to a classic Markovian model and (ii) can be only partially observed, through feedback relative to previous transmissions. We perform a thorough analytic study under four Ack/Nack feedback mechanisms, which to our knowledge represent all Ack/Nack feedback variations. Despite the general intractability of POMDPs, we prove that our communication service, under reliable feedback, can be inexpensively implemented. We precisely obtain a closed form solution specifying when to transmit over the channel, which allows to derive an energy-optimal dependable implementation. We show that an easy implementable structure for our communication service can also be obtained under lossy feedback, depending on the feedback mechanism.
\end{abstract}

\section{INTRODUCTION}

Unreliable asynchronous communication renders the design and analysis of distributed algorithms a challenging task [1]-[3]. Consequently, fault-tolerant distributed algorithms typically assume reliable or even synchronous (or partially synchronous) links [4]-[8]. However, in practice, message loss is unavoidable. In fact, all communication media are lossy to some extent, due to uncertainties stemming from various phenomena such as unpredictable system loads and physical properties of the media. For example, wireless and power line communication quality are influenced by path loss, fading, interference, switching of the power grid, activation of electrical equipment, etc [9]-[12]. Although message loss is common in wireless and power line networks, it actually exists everywhere [13], [14]. Due to the random occurrences of such inevitable phenomena, messages losses typically come and go over time. The communication between a pair of processes (abstracted by a communication link) thus experiences time-varying unreliability, i.e., changes in the quality of the communication link with time between lossy and reliable. In addition to being a problem by itself, time-varying unreliability induces asynchrony ${ }^{1}$, as successful message transmission delays become hard to anticipate.

The goal of this work is to mask such message losses through a communication service, that can be used by high level applications of the network. More precisely, we want to

\footnotetext{
*David Kozhaya

EPFL IC IIF LPD

INR 315 (Bâtiment INR) Station 14

CH-1015 Lausanne, Switzerland

${ }^{1}$ Asynchrony means here that there is no upper bound on message transmission delays, while synchrony means that the bound exists and is known.
}

provide a communication service which guarantees that message transfer, over a time-varying unreliable communication link, is: (i) always reliable and (ii) synchronous with high probability. Moreover, we want to design our communication service in an energy-efficient manner. In environments such as sensor networks [15]-[21], some devices have battery-powered wireless and recharging may be tedious or even impossible. In order to prolong the lifetime of a network, energy-efficient algorithms are crucial [22]-[30]. Distributed algorithms can thus transparently utilize this communication service to deliver energy-efficient services at the network level, e.g., to build an energy-efficient reliable broadcast or higher level abstracts.

To capture the time-varying message loss of a communication link, we consider a widely-used approach for such cases, the Gilbert-Elliot (GE) model [31], [32]. The GE model, consisting of two states (see Fig. 1), is a simple nontrivial finite state Markov chain (FSMC) [33], established to capture well message loss behaviour resulting from randomly occurring phenomena [34]-[36]. In fact, the GE model has been empirically verified, by a large body of work [32], [34], [37]-[40], as a good approximation of message losses in real-life communication scenarios. The GE model, for instance, has been used to model losses in wireless media IEEE 802.11 [40], wired power line networks [41] and other hybrid networks [13], [14]. The two states of the GE model (Fig. 1), noted by good and bad, can for example abstract the following: the communication link between a pair of processes occupies the bad state when the packet success-rate drops below a certain "unacceptable" threshold and the good state otherwise. The cause for these state transitions can, itself, lie in the random phenomena leading to message loss [34].

As in [38], [42]-[44], we assume that the current state of the link is not known to the sending process. The sender can however benefit from the feedback regarding previous transmissions to guess the current state. It can thus make better decisions of when to transmit; for example to avoid transmitting when the link state is bad. Such adaptive transmission decisions employ link prediction to appropriately adjust the transmission rate to the varying link conditions. In short, transmission policies which tell the sending process when to transmit and when to withhold from transmitting can be devised.

A reliable communication can be achieved by retransmitting a message until it has been received [45]. However, the rate at which the protocol attempts to retransmit yields a trade-off between (i) low energy consumption, (ii) high throughput and (iii) low latency. At one extreme, while merely optimizing for throughput and latency favors transmitting at every possible opportunity, this scheme results in maximum waste of energy. Especially at times when the link might be in 
a "bad condition", i.e., constantly losing messages, for a long duration. At the other extreme, optimizing solely for energy might lead to a throughput bottleneck and an overwhelming message latency. Given this trade-off, we address the question of how to build our communication service optimally by first studying the fundamental question of when to transmit.

In particular, we seek to solve the optimal transmission policy which defines when should a sender transmit messages in order to optimize a defined energy-throughput balance while favoring lower latency. We also address two corollary questions: (i) can optimal transmission policies guarantee reliability under all desired energy-throughput trade-offs? If not, under what energy-throughput trade-offs can reliability be ensured? (ii) how to provide synchronous guarantees with high probability, given unbounded time-varying message losses between a pair of processes?

Determining the optimal transmission policy in such a setting is an instance of partially observable Markov decision processes (POMDPs), known to be notoriously intractable [46]. We investigate the optimal transmission policy under various Ack/Nack feedback schemes ${ }^{2}$.

For presentation simplicity, we first conduct our analysis under a reliable feedback mechanism, where a sending process is notified about the success/failure of the previous transmission. We accomplish the following:

Optimal Transmission. Despite the fact that POMDPs are P-SPACE hard, we derive explicit solutions proving that the optimal transmission scheme, conforms to a computationally inexpensive and easily implementable structure.

Reliability. We show that the optimal transmission policy, under certain energy-throughput trade-offs and link parameters $^{3}$, can stop transmission for good. Suspending transmission as such prevents reliable communication. We hence identify the necessary conditions of system parameters to achieve reliability. We prove that when reliability is guaranteed, the optimal transmission policy takes one of three forms: constantly transmit, back-off on bad and skip if good.

Synchrony. Despite the dynamic message loss, we establish methods to obtain high probability guarantees on the total time required to successfully send a message over the link.

We then study the impact of lossy feedback (feedback which can be lost) on the optimal transmission by considering different feedback mechanisms. To the best of our knowledge, our work is the first to study optimal transmission under lossy feedback. Assuming a mechanism where feedback about the link state is periodically sent, we show that a myopic greedy policy [47] is optimal and reliable. If feedback is sent only regarding the times when the sender transmits over the link (regardless if the transmitted message is successfully received or not), we show that the optimal transmission will be similar to that in which feedback is reliable. In other words, closed form expressions allowing an easy implementation of the policy can be obtained. However, if we assume that the feedback is only sent when transmission is successful, we showcase that the optimal transmission policy does not necessarily comply with an easily implementable structure.

\footnotetext{
${ }^{2}$ To our knowledge, the feedback schemes we consider cover all possible Ack/Nack feedback mechanisms.

${ }^{3}$ An example could be when the energy cost relative to transmitting is very high and the link is rarely in the good state.
}

Contributions. Our paper shows how to provide, over a time-varying unreliable link, an energy-efficient, reliable communication that is synchronous with high probability.

We achieve energy-efficiency by solving the optimal transmission problem for time-varying links under incomplete information about link quality. In other words, we solve a decision problem (when to transmit messages) in a POMDP setting using the Gilbert-Elliot (GE) model to capture time-varying message losses over a communication link. Although the GE model admits a simple structure consisting of two states, it has been shown that POMDP approaches associated with the GE model are theoretically intractable (P-SPACE hard) to solve in various decision problem contexts [43], [46], [48]. Despite the difficulty associated with the incomplete link quality information, we derive in this paper explicit solutions for the optimal transmission problem and for the first time under multiple lossy feedback schemes. Our main contributions can be summarized as:

- A first full analytic study of optimal transmission policies of time-varying links for several reliable and lossy feedback.

- A derivation of explicit and closed form solutions to implement optimal transmission policies.

- An establishment of the necessary conditions for reliable communication using optimal transmission policies.

- Methods to obtain synchronous message transfers with high probability.

Road-map. The rest of the paper is organized as follows: Section II discusses the related work. Section III describes the system model, the various feedback mechanisms and demonstrates a way of defining an energy-throughput balance. Section IV formulates the problem of determining the optimal transmission policy. In Sections V and VI we prove that the optimal transmission strategy under reliable feedback has an implementable structure, provide respective closed form expressions and determine the necessary conditions and transmission forms guaranteeing reliability. In Section VII we study the impact of lossy feedback on the optimal transmission scheme. In Section VIII we show how to achieve a synchronous behaviour over the link, with a high probability. We conclude the paper in Section IX.

\section{RELATED WORK}

In this section, we discuss related work on (i) reliable links and dynamic link failures in distributed algorithms, (ii) communication over time-varying links under POMDP settings and (iii) restless bandits.

Previous work on distributed algorithms addressed the issue of achieving reliable communication over lossy links at different levels [?], [49], [50]. For example Aguilera et al. implemented a failure detector allowing a quiescent reliable communication when processes can fail [49]. Guerraoui et al. defined the stubborn link abstraction which is weaker than a reliable link but strong enough to solve important distributed problems such as consensus [50]. Another work by Basu et al. studied the solvability of problems in a system with process crashes and message loss [?]. Aside from reliable communication, certain distributed algorithms approaches studied systems with dynamic communication failures. Multiple efforts [51], [52] addressed the $k$-consensus problem, which requires only $k$ processes to eventually decide. Moniz et al. [51] considered a 
system where message transmissions can be faulty: after some unknown time at most $\left\lceil\frac{n}{2}\right\rceil(n-k)+k-2$ faulty transmissions occur at each round. The number of faults per round prior to this is unrestricted. In a different setting, Moniz et al. [52] considered a communication system where a process sending a message will send it to all other processes sharing that link. Their algorithm tolerates up to $f$ Byzantine processes and requires the number of omission faults affecting correct processes to be at most $\left\lceil\frac{n-f}{2}\right\rceil(n-k-f)+k-2$ in infinitely many rounds, a fairness assumption to guarantee liveness. The work in this paper goes one step further as it tries to achieve energy-optimal algorithms. Also, in comparison with previous work accounting for links failures through omission faults, our work does not specify any bounds on the number of message losses and allows, through a probabilistic loss behaviour, to send messages intelligently, precisely avoiding potential losses.

In a different context, existing work applied tools from Markov decision processes to solve communication problems over time-varying links, e.g., [37], [38], [53]. In their work, Laourine and Tong considered actions with a variable number of bits being sent in each action [38]. The problem we investigate in this paper is different. We consider transmission with a fixed number of bits in all actions and optimize for throughput under energy costs, which is not accounted for in Laourine and Tong's work [38]. Another work by Johnston and Krishnamurthy, applied results from optimal search theory of a Markovian target to find optimal transmission strategies [53]. They studied the problem of transmitting a single file/message over the link maximizing the average reward. In contrast, we consider an infinite sequence of messages to be transmitted and optimize for the discounted reward, which allows to favor lower latency. Most related to this paper is the work by Zhang and Wassermanin [37], which targeted achieving a suitable balance between throughput and energy consumption. The authors proved that the optimal transmission policy follows a threshold structure. Their paper assumed multiple power levels for transmission where a sender must decide on one, in case it decides to send. This energy level itself affects the probability of the message content being delivered correctly. However, in this paper we assume that a link can lose messages but it does not manipulate their content. Besides, we define the optimal policy in terms of the costs and we obtain closed form solutions in terms of the system parameters. It is also important to note that all the above mentioned work have mainly considered positively correlated links (see Section V) and error-free feedback. To the best of our knowledge, this paper is thus the first to analyse all cases of link memory, i.e., memoryless, positively and negatively correlated cases and to study optimal transmission under lossy feedback.

Another closely related area to the problem studied in this paper is that of restless multi-armed bandits that was applied mainly to cognitive radio networks [43], [44], [47]. In restless multi-armed bandits, there is a set of $N$ independent projects that evolve over time and can yield some reward once they are activated based on their state at that time. A player is required to activate one of these projects at each time in such a way that maximizes the total long-term expected reward. The problem was studied in a setting where projects evolve according to the GE model where the parameters are known [43], [44], [47]. Zhao et al. prove that when projects are independent and identically distributed, a myopic policy, which activates the

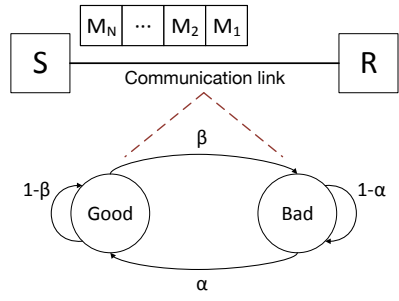

Fig. 1. A time-varying communication link under the 2-state GE model

project with the highest belief, is optimal under the positive correlation assumption [44]. Another work by Guha et al. also studied this problem under the positive correlation assumption and independent but not necessarily identically distributed links [43]. They propose an approximation algorithm with a performance guarantee of 2. Among the work on restless bandits, that by Liu and Zhao is perhaps the closest to the work in this paper [44]. The authors compute a closed form of an index known as the Whittle index (see [48] for a complete definition of this index), which measures the attractiveness of activating a project. They propose to activate at every time the project with the highest index. The work in this paper uses a notion similar to the subsidy of the Whittle index to derive its closed forms. However, the link considered here has nontrivial differences from the link considered in all cited work on restless bandits [43], [44], [47], as we permit for feedback to be lost. We also allow for positive rewards and negative costs to coexist and allow for costs to be incurred at two levels: when the link is idled and when a transmission fails.

\section{SySTEM MODEL}

We study the communication between any two processes. One process, noted by $S$, needs to send messages to the other process noted by $R$ (see Figure 1). We assume discrete time events denoted by $T_{\text {sys }}=\left\{t_{1}, t_{2}, t_{3}, \ldots\right\}$. A subset, $\mathcal{T}$, of these time events occur at $S$, where $t_{i} \in \mathcal{T} \forall i$ odd (i.e., $\left.\mathcal{T}=\left\{t_{1}, t_{3}, t_{5}, \ldots\right\}\right)$. The time interval between consecutive events in $T_{\text {sys }}$ is an upper bound on the propagation delay over the channel in a single direction. As a result, the time interval between consecutive time events in $\mathcal{T}$ is an upper bound on the round trip propagation delay over the channel. We designate by the time events in $\mathcal{T}$ the instances at which $S$ is allowed to use the channel, if it desires.

As shown in Figure 1, the communication channel conforms to a 2-state Gilbert-Elliot (GE) model. At any point in time, the channel can be in one of the two states: the good state or the bad state. The channel transitions with time, i.e., the channel moves to its new state, which can be the same state it existed in or the other state. The time instants at which the channel transitions are known as the transition times of the channel. For example, given the channel is in the good state at some point in time, it will remain in the same state at the next transition time with probability $1-\beta$ and will move to the other state with probability $\beta$. Similarly if the channel state is bad at some point in time, it will remain bad at the next transition time with probability $(1-\alpha)$ and will shift to good with probability $\alpha$. The channel state remains fixed in the interval separating the transition times. We assume that $S$ 
knows the parameters of the channel, i.e., $\alpha$ and $\beta$ (in practice $S$ can estimate these parameters, see e.g., [54]).

If $S$ sends a message $m$ at some time $t_{i} \in \mathcal{T}$ and the channel is in the good state in the interval $\left[t_{i}, t_{i+1}[\right.$, then $m$ is received by $R$ at time $t: t_{i}<t<t_{i+1}$ (i.e., before $t_{i+1}$ ). We say $R$ receives $\boldsymbol{m}$ by time $t_{i+1}$. If however the channel state is bad, the transmission of message $m$ fails, in which case $S$ will retry to send this same message $m$ in the following time unit in $\mathcal{T}$. Consequently, transmitting a message might span several time units (depending on the state of the channel). Meanwhile, new messages (whether from outside or generated by $S$ itself) that may arrive to $S$ will be enqueued. We assume a FIFO queue where a message is dequeued only when it is successfully acknowledged. If a message transmission fails, the respective message remains at the top of the queue. Practically, this queue should be of a finite size. We thus assume that the rate at which new messages arrive to $S$, relative to the rate at which messages are dequeued, amounts to having at all times a non-empty queue ${ }^{4}$ of size at most $N$. In practice and with the help of queuing theory, $N$ can be chosen such that the probability of the queue overflowing is extremely small ( $\approx$ negligible). Guaranteeing a non-empty queue can be achieved by having $S$ generate dummy messages whenever needed, which then serve to probe the channel state.

We investigate the optimal transmission scheme, i.e. what time instances in $\mathcal{T}$ it is optimal for $S$ to transmit a message over the channel, under four Ack/Nack feedback mechanisms. These four feedback mechanisms constitute, to the best of our knowledge, all possible variations of an Ack/Nack feedback mechanism.

Perfect Feedback. This feedback mechanism allows the sender to know what was the channel state in the last carried transmission. This mechanism is analogous to sensing the channel, assuming error-free sensing. To implement this feedback scheme, we assume that the channel transitions at every time instant in $\mathcal{T}$ only. When $S$ sends a message $m$ at some time $t_{i} \in \mathcal{T}$ and the channel is in the good state, $m$ will be successfully propagated to $R$. $R$ then directly replies with an acknowledgment, ack, which will be received by $S$ before the next time instant in $\mathcal{T}$, i.e., $t_{i+2}$. However, if the channel state is bad, transmission fails and $R$ receives nothing. $S$ is thus informed about the success of the last message transmission, and simultaneously about the last channel state, by the presence or absence of an acknowledgment from $R$.

Constant Feedback. In this mechanism, the sender expects to receive periodical feedback from the receiver about the channel state. However, this feedback can be lost. This mechanism is analogous to sensing the channel on a periodical basis. We achieve this feedback by assuming that the channel transitions at every time instant in $T_{\text {sys }}$. We also assume that $R$ has access to the time instants $\mathcal{T}_{r c v}=\left\{t_{2}, t_{4}, t_{6}, \ldots\right\} . R$ thus sends a message to $S$ at every time instant in $\mathcal{T}_{r c v}$ regardless if $S$ has sent something or not. The message sent by $R$ is an ack if some message from $S$ is received and is a nack otherwise. As a result, messages sent by $R$ can be lost independently of those sent by $S$.

Smart Feedback. In this mechanism, the sender expects feedback, despite potential losses, every time it transmits

\footnotetext{
${ }^{4}$ This is otherwise known as the infinite backlog assumption resulting in infinite messages to send.
}

over the channel. This mechanism is analogous to error-free sensing, however in a lossy environment. Smart feedback can be achieved similar to constant feedback, except that we consider now that $R$ knows the times at which $S$ sends a message. As such $R$ will not send anything when it's not expecting to receive a message from $S$. The assumption that $R$ knows the sending times of $S$ can be easily satisfied under deterministic sending schemes, e.g., constantly transmitting over the channel.

Unreliable Feedback. The sender in this feedback mechanism is not sure if it can obtain feedback even when it transmits over the channel. This mechanism is analogous to a sensing service which is not available all the time. To implement this mechanism, we also assume that the channel transitions at every time instant in $T_{\text {sys }}$. As noted in constant feedback, having the transition times in $T_{\text {sys }}$ means that messages sent by $R$ can be lost independently of those sent by $S$. The receiver $R$ sends an ack message to $S$ only when it receives a message from $S$; otherwise $R$ sends nothing.

At each time instant in $\mathcal{T}, S$ can either (i) use the channel to transmit a message or (ii) idle transmission. Both transmission and idling incur energy costs. We assume that a message transmission incurs a cost (negative reward) of $c_{p}(\leq 0)$, while idling incurs a cost of $c_{d}(\leq 0)$. It is obvious that in practice $c_{p}<c_{d}$ (since the idling energy cost is at least one order of magnitude less than sending in wireless sensor networks, see e.g., [55]), otherwise the optimal policy would be to always transmit. If a message is both transmitted and acknowledged successfully, $S$ obtains an additional reward $r_{s}>\left|c_{p}\right|$. In this case, the total reward relative to a successful transmission is $r=r_{s}+c_{p}(>0)$, while an unsuccessful or unacknowledged transmission gives no additional reward. This assignment of rewards and costs constitutes a generic function which allows to define any desired weighted balance between energy and throughput.

Illustration of Cost Assignment. Let us set the idling cost $c_{d}$ to 0 and try to establish a throughput-energy balance based on the reward $r_{s}$ and cost $c_{p}$. Assuming that messages sent by $S$ are of maximum size, i.e., equivalent to the channel capacity, then a reward of value $r_{s}=1$ can be assigned. This reflects a maximum throughput, achieved by utilizing $100 \%$ of the channel capacity in every time unit where transmission is successful. The amount of energy needed to transmit these fixed size messages is possible to obtain by measuring how much power is dissipated in the required transmission period. Assuming that $S$ is a battery operated process ${ }^{5}$, we can calculate the average percentage energy consumption relative to a single transmission (guaranteed to be $<1$ ), which can in turn be assigned as the value of the energy cost $c_{p}$.

\section{Optimal TRANSMission Formulation}

In this section, we formally define our first question concerning how our communication service can be made energy optimal. In other words, we define mathematically the problem of determining when to transmit over the channel so that a defined cost function is optimized. For simplicity we consider throughout the rest of the paper $t=\left\{t_{i}: t_{i} \in \mathcal{T}\right\}$ and

\footnotetext{
${ }^{5}$ Wireless sensor networks in a variety of contexts such as building automation and smart grids have finite energy sources or intermittent energy harvesting scenarios.
} 
$t+1=t_{i+2}$, i.e., the next time instant in $\mathcal{T}$. Let $a_{t}$ be the action taken a time $t . a_{t}=1\left(a_{t}=0\right)$ corresponds to transmitting (idling) at time $t$ respectively. The transmission policy, $\pi$, will then be the set of all decisions to be taken, i.e., $a_{t} \forall t$. We denote by $o_{t}$ the feedback received by $S$ (precisely from $R$ ) by time $t$. If nothing is received by time $t$ then $o_{t}=\perp$. Let $\mathcal{R}\left(a_{t}, o_{t+1}\right)$ be the reward obtained at time $t$ relative to action $a_{t}$ and the corresponding feedback relative to action $a_{t}$ (which is obtained by time $t+1$ ).

$$
\mathcal{R}\left(a_{t}, o_{t+1}\right)= \begin{cases}r & a_{t}=1, o_{t+1}=a c k \\ c_{p} & a_{t}=1, o_{t+1}=\text { nack } \vee \perp, \\ c_{d} & a_{t}=0\end{cases}
$$

Under all feedback mechanisms, $S$ can make probabilistic guesses about the link state. Consequently, a conditional probability that the link state is good given the last received feedback from $R$, can be maintained by $S$ at all times in $t \in \mathcal{T}$. This conditional probability is called the link belief $w_{t}$. We compute the link belief under each feedback mechanism.

Perfect Feedback. Under perfect feedback, if $S$ sends a message at time $t$ then at time $t+1, S$ will know what was channel state in $[t, t+1$ [. Accordingly the channel belief is updated at the end of every time $t \in \mathcal{T}$ as follows:

$$
w_{t+1}= \begin{cases}1-\beta & a_{t}=1, o_{t+1}=a c k \\ \alpha & a_{t}=1, o_{t+1}=\perp \\ \tau\left(w_{t}\right)=(1-\beta) w_{t}+\alpha\left(1-w_{t}\right) & a_{t}=0\end{cases}
$$

Constant Feedback. At all times $t \in \mathcal{T}, S$ can know the exact last state of the channel before $t$. Consequently, the channel belief $w$ is updated at every time $t$ as follows:

$$
w_{t+1}= \begin{cases}1-\beta & o_{t+1}=\text { ack } \vee \text { nack }, \\ \alpha & o_{t+1}=\perp,\end{cases}
$$

Smart Feedback. $S$ can know the exact previous state of the channel at all times $t: a_{t}=1$, i.e., the times at which $S$ sends a message over the channel. Consequently, the channel belief $w$ is updated at time instants $t$ as follows:

$$
w_{t+1}= \begin{cases}1-\beta & a_{t}=1, o_{t+1}=a c k \vee \text { nack } \\ \alpha & a_{t}=1, o_{t+1}=\perp, \\ \tau^{2}\left(w_{t}\right)=\tau\left(\tau\left(w_{t}\right)\right) & a_{t}=0 .\end{cases}
$$

Unreliable Feedback. $S$ can know the exact previous state of the channel at all times $t: a_{t}=1 \wedge o_{t+1}=a c k$, i.e., the times at which $S$ sends a message and receives an acknowledgement for that message. At all other times $S$ can not be sure about the previous channel state. Consequently, the channel belief $w$ is updated at time instants $t$ as follows:

$$
w_{t+1}= \begin{cases}1-\beta & a_{t}=1, o_{t+1}=a c k \\ \mathrm{~T}\left(w_{t}\right)=\frac{\tau(\alpha)-\alpha w_{t}(2-2 \beta-\alpha)}{1-w_{t}(1-\beta)} & a_{t}=1, o_{t+1}=\perp, \\ \tau^{2}\left(w_{t}\right)=\tau\left(\tau\left(w_{t}\right)\right) & a_{t}=0 .\end{cases}
$$

We now show how $\mathrm{T}\left(w_{t}\right)$ is derived. By Bayes' Theorem,

$$
\begin{aligned}
\mathrm{T}\left(w_{t}\right) & =\operatorname{Pr}\left(s_{t+1}=G \mid w_{t}, a_{t}, o_{t+1}=\perp\right) \\
& =\frac{\operatorname{Pr}\left(s_{t+1}=G, w_{t}, a_{t}, o_{t+1}=\perp\right)}{\left.\operatorname{Pr}\left(o_{t+1}=\perp\right) \mid w_{t}, a_{t}\right)}
\end{aligned}
$$

where $s_{t}$ is the channel state at time $t$ and

$$
\begin{gathered}
\operatorname{Pr}\left(s_{t+1}=G, w_{t}, a_{t}, o_{t+1}=\perp\right) \\
=\sum_{s_{t}} \operatorname{Pr}\left(s_{t+1}=G \mid s_{t}, a_{t}, o_{t+1}=\perp\right) \operatorname{Pr}\left(o_{t+1}=\perp \mid s_{t}, a_{t}\right) \operatorname{Pr}\left(s_{t}, w_{t}\right) \\
\mathrm{T}\left(w_{t}\right)=\frac{\tau(\alpha)-\alpha w_{t}(2-2 \beta-\alpha)}{1-w_{t}(1-\beta)}
\end{gathered}
$$

We want to favor lower message latency while maximizing the defined energy-throughput cost function (Section I), i.e., we consider a delay sensitive communication. Accordingly, the performance measure we seek to maximize is the expected total discounted reward. The discounting factor is a constant denoted by $\gamma$, such that $0<\gamma<1$. This $\gamma$ can be roughly thought of as a penalty for delay. For practical choice of $\gamma$, note that $\gamma$ weights future rewards. Thus, a smaller $\gamma$ should be chosen for more delay-sensitive applications, as it puts more emphasis on early transmissions. The expected total discounted reward can be formally written as:

$$
E_{\pi}\left[\sum_{j=0}^{\infty} \gamma^{j} \mathcal{R}\left(a_{t_{j+1}}, o_{t_{j+3}}\right) \mid w_{0}\right]
$$

where $w_{0}$ is the initial belief. The objective is to obtain the maximum expected total discounted reward that can be incurred from transmitting over a single channel, also known as the value function $V_{\gamma}(w)$. Let $V_{\gamma}(w ; a=1)$ (and analogously $\left.V_{\gamma}(w ; a=0)\right)$ designate the expected total discounted reward from transmitting (not transmitting) on the channel in the first decision followed by the optimal decisions in future times. Due to POMDP theory, the value function satisfies the Bellman equation and thus $V_{\gamma}(w)$ can be written as [38]

$$
V_{\gamma}(w)=\max \left\{V_{\gamma}(w ; a=1), V_{\gamma}(w ; a=0)\right\} .
$$

\section{Optimal Transmission StRategies}

For presentation simplicity we first derive the value function and study the optimal transmission policy under the perfect feedback mechanism. We study the optimal transmission policy under the other feedback mechanisms later in Section VII.

Transmitting over the link yields an immediate expected reward of:

$$
\begin{aligned}
w \mathcal{R}\left(a_{t}=1, o_{t+1}=a c k\right) & +(1-w) \mathcal{R}\left(a_{t}=1, o_{t+1}=\perp\right) \\
& =w r+(1-w) c_{p}=w\left(r-c_{p}\right)+c_{p} .
\end{aligned}
$$

The future maximum expected total discounted reward, relative to transmitting over the channel, will be either: (i) $\gamma V_{\gamma}(1-\beta)$ (if the current state is good) or (ii) $\gamma V_{\gamma}(\alpha)$ (if the current state is bad). The former occurs with probability $w$ while the latter occurs with probability $1-w$ resulting in:

$V_{\gamma}(w ; a=1)=w\left(r-c_{p}\right)+c_{p}+\gamma\left[w V_{\gamma}(1-\beta)+(1-w) V_{\gamma}(\alpha)\right]$.

Idling however yields an immediate expected cost of $c_{d}$ (since no other cost/reward exists relative to idling the channel). By the update function of the channel belief (Section IV), $w$ deterministically evolves to $\tau(w)$ as a result of not using the channel. The consequent future maximum expected total discounted reward is $V_{\gamma}(\tau(w))$ occurring with probability 1 . Hence, $V_{\gamma}(w ; a=0)=c_{d}+\gamma V_{\gamma}(\tau(w))$. 
The value function, $V_{\gamma}(w)$, can be recursively written as:

$V_{\gamma}(w)=\max \left\{V_{\gamma}(w ; a=1), V_{\gamma}(w ; a=0)\right\}$

$=\max \left\{w\left(r-c_{p}\right)+c_{p}+\gamma\left[w V_{\gamma}(1-\beta)+(1-w) V_{\gamma}(\alpha)\right], c_{d}+\gamma V_{\gamma}(\tau(w))\right\}$,

We distinguish between the different channel types and determine the optimal transmission strategy in each case. The channel can be categorized, based on its transition probabilities, as either being memoryless or not. The latter itself is subdivided into two categories: positively correlated $(1-\beta>\alpha)$ and negatively correlated $(1-\beta<\alpha)$.

Memoryless Channel. The channel is memoryless when the probability of being in either state at the next time step is independent from current state, i.e. $1-\beta-\alpha=0$. As a consequence, $1-\beta=\alpha=\tau(w)=p$. The value function in (5), thus reduces to

$$
\begin{aligned}
V_{\gamma}(w) & =\max \left\{w\left(r-c_{p}\right)+c_{p}+\gamma V_{\gamma}(p), c_{d}+\gamma V_{\gamma}(p)\right\} \\
& =\max \left\{w\left(r-c_{p}\right)+c_{p}, c_{d}\right\}+\gamma V_{\gamma}(p) .
\end{aligned}
$$

The optimal transmission policy hence depends merely on the values of $w\left(r-c_{p}\right)+c_{p}$ and $c_{d}$ :

$$
\text { optimal policy }= \begin{cases}\text { transmit } & \text { if } w>\frac{c_{d}-c_{p}}{r-c_{p}} \\ \text { idle } & \text { otherwise. }\end{cases}
$$

Since $1-\beta=\alpha=\tau(w)=p, w$ will have a constant value for a given channel. The optimal policy thus is either: (i) transmit on the channel at every $t$ or (ii) never transmit on the channel.

Channel with Memory. It is well established that the value function can be obtained by value iteration as a uniform limit of cost functions for finite horizon problems, which are continuous, piecewise linear and convex [42], [56]. The uniform convergence follows from the discounted dynamic operator being a contraction mapping [56]. As a consequence of uniform convergence, $V_{\gamma}(w)$ is a convex function in $w$ continuous on $[0,1]$.

Lemma 1: If $c_{d}<w\left(r-c_{p}\right)+c_{p}$ (in particular if $c_{d}<c_{p}$ ), then the optimal decision is to use the channel for transmission at every $t$.

Proof: By convexity of $V_{\gamma}(w)$ in $w$ we have:

$V_{\gamma}(\tau(w))=V_{\gamma}(w(1-\beta)+\alpha(1-w)) \leq w V_{\gamma}(1-\beta)+(1-w) V_{\gamma}(\alpha)$ $V_{\gamma}(w ; a=1)$ will be greater than $V_{\gamma}(w ; a=0)$, if $c_{d}<$ $w\left(r-c_{p}\right)+c_{p}$, i.e., if $c_{d}-c_{p}<w\left(r-c_{p}\right)$.

Given that $w\left(r-c_{p}\right) \geq 0, V_{\gamma}(w ; a=1)>V_{\gamma}(w ; a=0)$ if $c_{d}-c_{p}<0$.

Lemma 2: For a channel with a defined cost function, there exists a unique value $w^{*}$ such that $V_{\gamma}\left(w^{*} ; a=1\right)=$ $V_{\gamma}\left(w^{*} ; a=0\right), V_{\gamma}(w ; a=1)<V_{\gamma}(w ; a=0) \forall w<w^{*}$ and $V_{\gamma}(w ; a=1)>V_{\gamma}(w ; a=0) \forall w>w^{*}$.

Proof: At $w=0$ :

$V_{\gamma}(0 ; a=1)=c_{p}+\gamma V_{\gamma}(\alpha)$ and $V_{\gamma}(0 ; a=0)=c_{d}+\gamma V_{\gamma}(\alpha)$. From Lemma $1, c_{d}<c_{p}$ trivializes the optimal policy to that which constantly transmits over the channel. We thus consider $c_{d}>c_{p}$ which yields $V_{\gamma}(0 ; a=1)<V_{\gamma}(0 ; a=0)$.

At $w=1$ :

$V_{\gamma}(1 ; a=1)=r+\gamma V_{\gamma}(1-\beta)$ and $V_{\gamma}(1 ; a=0)=c_{d}+$ $\gamma V_{\gamma}(1-\beta)$. Since $r>0$ and $c_{d} \leq 0$, we get $V_{\gamma}(1 ; a=1)>$ $V_{\gamma}(1 ; a=0)$.

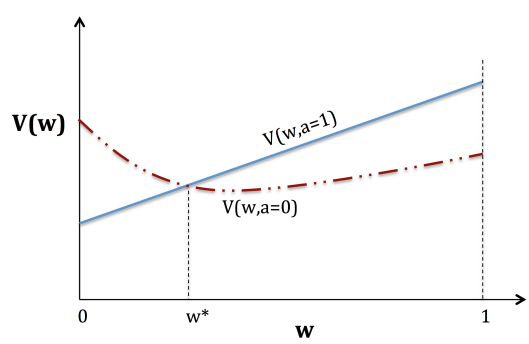

Fig. 2. The behaviour of the value functions for transmitting and idling.

It can be seen that $V_{\gamma}(w ; a=1)$ is linear in $w$. Following from the convexity of $V_{\gamma}(w)$, we can conclude that $V_{\gamma}(w ; a=0)$ is convex in $w$. As a result, there exists a single intersection point between $V_{\gamma}(w ; a=1)$ and $V_{\gamma}(w ; a=0)$, where the implication $w^{*}$ is unique comes from. This leads to the graph shown in Figure 2 concluding the proof.

As a direct consequence of Lemma 2 the optimal policy has the following structure:

$$
\text { optimal policy }= \begin{cases}\text { transmit } & \text { if } w>w^{*} \\ \text { idle } & \text { if } w<w^{*}\end{cases}
$$

We compute the value of $w^{*}$ by distinguishing between positively and negatively correlated channels. Depending on the possible position of $w^{*}$ with respect to $\alpha, 1-\beta$ and $\pi_{g}$ (the stationary probability of being in the good state, $\pi_{g}=\frac{\alpha}{\alpha+\beta}$ ), $w^{*}$ takes different values and admits different closed form expressions (refer to Appendix A). However, this requires first to guess the position of $w^{*}$ with respect to $\alpha, 1-\beta$ and $\pi_{g}$ for a given channel and given cost assignment. Not knowing the position of $w^{*}$ in the general case, we define the optimal policy in terms of costs. The computed closed form expressions of $w^{*}$, for all cases, depend on the cost $c_{d}$. More precisely, these closed forms show that $w^{*}$ is strictly increasing in $c_{d}$ (refer to Appendix A). We substitute the given fixed cost $c_{d}$ by an unknown cost $C(w)$. We let $C(w)$ be the cost such that $w^{*}=w$. In other words, $C(w)$ is the idling cost under which $V_{\gamma}(w ; a=1)=V_{\gamma}(w ; a=0)$.

Lemma 3: There exists a unique cost $C(w)$ such that $V_{\gamma}(w ; a=1)=V_{\gamma}(w ; a=0)$, where

$$
\begin{gathered}
V_{\gamma}(w ; a=1)=w\left(r-c_{p}\right)+c_{p}+\gamma\left[w V_{\gamma}(1-\beta)+(1-w) V_{\gamma}(\alpha)\right], \\
V_{\gamma}(w ; a=0)=C(w)+\gamma V_{\gamma}(\tau(w)) .
\end{gathered}
$$

Proof: By Lemma 2 there is a unique intersection point $\left(w^{*}\right)$ between $V_{\gamma}\left(w^{*} ; a=1\right)$ and $V_{\gamma}\left(w^{*} ; a=0\right)$. Since $w^{*}$ is strictly increasing in the idling $\operatorname{cost} c_{d}$ (refer to Appendix A), then no two or more distinct idling costs can lead to the same $w^{*}$, which concludes the proof.

The closed form expressions of $C(w)$ can then be obtained by simply inverting the closed form expressions of $w^{*}$ and setting $w^{*}=w$ (see Figure 3).

Lemma 4: $C(w)$ is strictly increasing in $w$.

Proof: From Appendix A, $w^{*}$ is strictly increasing in $c_{d}$, which means that $c_{d}$ is also strictly increasing in $w^{*}$ (inverting the relation preserves the monotonicity). But the expressions for $C(w)$ are obtained by replacing $c_{d}$ by $C(w)$ and setting $w^{*}=w$, which concludes the proof. 


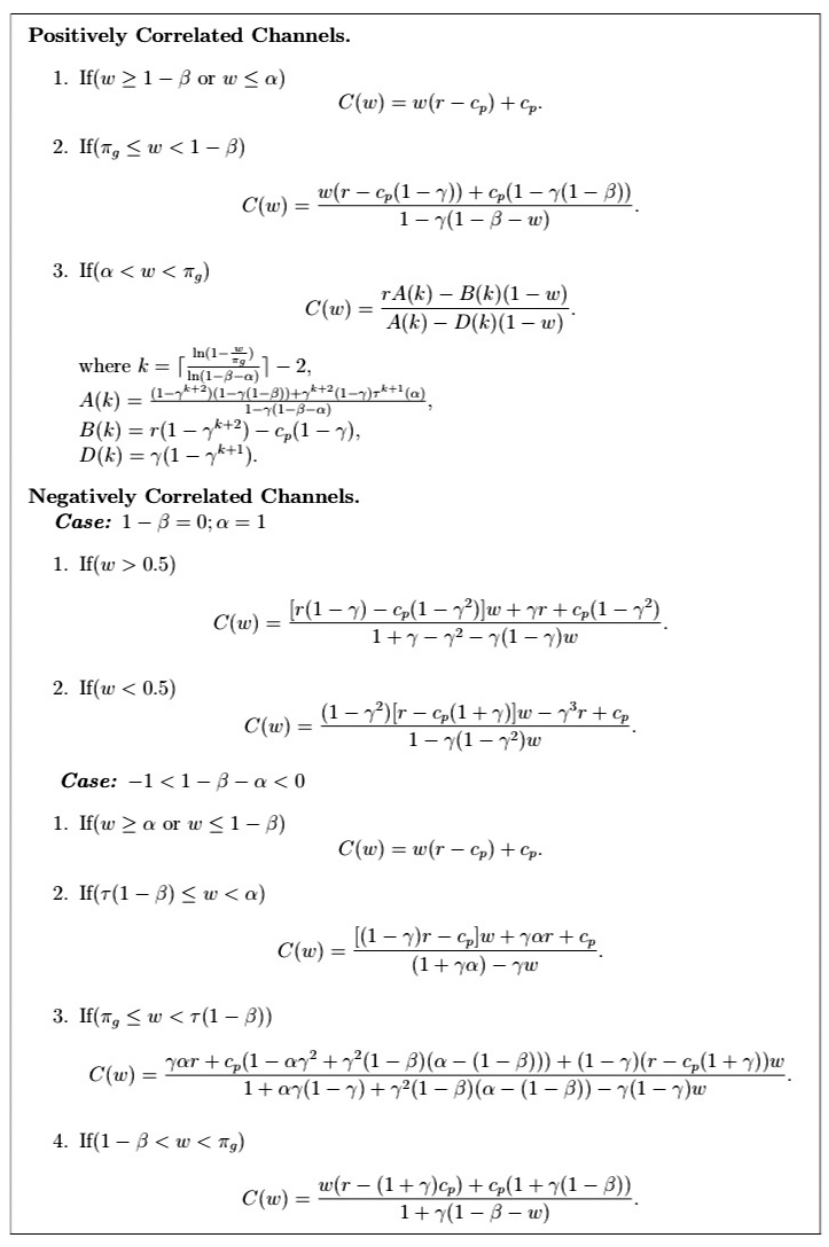

Fig. 3. Closed form expressions for $C(w)$.

Theorem 1: The optimal policy for a channel with memory under a given cost assignment is:

$$
\text { optimal policy }= \begin{cases}\text { transmit } & \text { if } c_{d}<C(w), \\ \text { idle } & \text { if } c_{d} \geq C(w) .\end{cases}
$$

Proof: For a channel with a given idling $\operatorname{cost} c_{d}$, there exists by Lemma 2 a unique value $w^{*}$ which makes the action of transmitting on the channel as equally attractive as that of idling transmission. More precisely $C\left(w^{*}\right)=c_{d}$. By Lemma 4 , $C(w)>c_{d}$ for $w>w^{*}$. The optimal policy definition says to transmit if $w>w^{*}$ and idle otherwise. Thus $C(w)>c_{d}$ amounts to having the action of transmitting over the channel as optimal. Similarly by Lemma $4, C(w)<c_{d}$ for $w<w^{*}$, which means that idling transmission is optimal.

\section{Optimal Reliable Transmission}

Still considering perfect feedback, we investigate in this section how to guarantee reliability using optimal transmission schemes. Clearly reliability is guaranteed if and only if optimal decisions do not suspend transmission endlessly.

Lemma 5: Optimal transmission policies do not always guarantee reliability across a channel be it memoryless or not.

Proof: We prove the lemma for each case of channel memory by illustrating a counter example showing endless suspension of transmission under optimal decisions.

Memoryless. In the memoryless case the optimal decision at every time instant $t$ is transmit only if $w>\frac{c_{d}-c_{p}}{r-c_{p}}$; however $w=p=1-\beta=\alpha, \forall t>0$. An assignment of $c_{d}=0$ and $r=-c_{p}$ leads to $\frac{c_{d}-c_{p}}{r-c_{p}}=0.5$. Hence any channel satisfying $1-\beta=\alpha<0.5$ will have the optimal decision of always idling the channel.

Positively correlated. One possible cost assignment could lead to the following relation being satisfied: $c_{d} \geq(1-\beta)(r-$ $\left.c_{p}\right)+c_{p}$. Such an assignment can happen in cases where the channel rarely resides in the good state and the energy costs are relatively high (e.g. $c_{d}=0, r=-c_{p}$ and $1-\beta=0.3$ ). The expected reward relative to transmitting on the channel is $w\left(r-c_{p}\right)+c_{p}$. Note that $1-\beta \geq w \geq \alpha$ (a direct consequence of the belief update function in Section IV) and that $w\left(r-c_{p}\right)+c_{p}$ increases monotonically as $w$ increases. The expected reward $\left(c_{d}\right)$ relative to idling the channel is thus always greater than the maximum expected reward relative to using the channel. Hence attempting to transmit at any time will only make the value of the total expected discounted reward less.

Negatively correlated. As in the positively correlated case, a channel which rarely resides in the good state and whose energy costs are relatively high (e.g. $c_{d}=0, r=-c_{p}$ and $\alpha=0.3)$ may lead to having $c_{d} \geq \alpha\left(r-c_{p}\right)+c_{p}$ satisfied. Due to negative correlation, we have $1-\beta \leq w \leq \alpha$ (following from the belief update function in Section IV). Attempting to transmit on the channel at any time will yield an expected reward less than that obtained by idling the channel. This makes the decision of idling the channel at all times lead to the maximum value of the total expected discounted reward.

Theorem 2: In a memoryless channel, i.e., a channel with a constant probability, $1-p$, of losing messages, reliability is guaranteed under an optimal policy only if $p>\frac{c_{d}-c_{p}}{r-c_{p}}$. The policy in this case is to constantly transmit on the channel.

Proof: The proof follows directly from the optimal policy of memoryless channels in Section V.

Theorem 3: Given that a positively correlated channel is used at some time $t$, there are exactly 2 forms of optimal transmission policies at $[t+1, \ldots, \infty[$, capable of guaranteeing reliability.

1) Constantly Transmit: In case of high idling cost, keep sending, irrespective of the predicted channel state $\left(a_{t+i}=1 \forall i\right)$. This policy is optimal if $c_{d}<$ $\alpha\left(r-c_{p}\right)+c_{p}$.

2) Back-off on Bad: Transmit as long as the observed channel state is good. When the observed state is bad, transmission is withheld for $\mathrm{T}$ time instants (somehow wait until channel is expected to transition to the good state) after which transmission resumes again.

$$
\mathbf{T}=\left\lfloor\frac{\ln \frac{\left(r-c_{d}\right) A(k)+\left(1-w_{0}\right)\left(c_{d} D(k)-B(k)\right)}{w_{0}(B(k)-D(k))}}{\ln (1-\beta-\alpha)}\right\rfloor .
$$

This policy is optimal if $c_{d}<(1-\beta)\left(r-c_{p}\right)+c_{p} \wedge$ $c_{d} \geq(\alpha)\left(r-c_{p}\right)+c_{p}$. 
Proof: We split the possible search space of $w^{*}$, i.e. $[0 ; 1]$, into different regions. If $w^{*} \in\left[\pi_{g}, 1\right]$, and the channel is observed in the bad state, i.e., $w=\alpha$, then the optimal policy will be to suspend transmission forever. This follows from the fact that (14) and (15) lead to $w \geq w^{*}$ never being satisfied. The only possible range for $w^{*}$, such that transmission is never suspended forever, is to exist in the region $\left[0, \pi_{g}\right]$. This range can be split in two: $[0, \alpha] \cup\left[\alpha, \pi_{g}\right]$. Following from the update function of Section IV the channel belief eventually abides by:

$$
1-\beta \geq w \geq \alpha .
$$

If $w^{*} \in[0, \alpha]$, then by (6), $w>w^{*}$ will always be satisfied and the optimal policy would be to always use the channel. If $w^{*} \in\left[\alpha, \pi_{g}\right]$ then as long as the channel is observed to be $\operatorname{good}\left(w=1-\beta>w^{*}\right)$ it would be optimal to use the channel again. However if the channel is observed to be bad $\left(w=\alpha \leq w^{*}\right)$ then it is optimal not to use the channel until $w>w^{*}$ is satisfied, which by (14) happens in finite time T. By Theorem $1 w>w^{*}$ for $w^{*} \in\left[\alpha, \pi_{g}\right]$ is equivalent to $c_{d}<\frac{r A(k)-B(k)(1-w)}{A(k)-D(k)(1-w)}$, where $w=\tau^{\top}(\alpha)$ for $\mathrm{T} \in[0, \infty]$.

Theorem 4: Given a negatively correlated channel is used at some time $t$, there are exactly 2 forms for the optimal transmission policies at times $[t+1, \ldots, \infty]$, which are capable of ensuring reliability:

1) Constantly Transmit: This policy is optimal if $c_{d}<$ $(1-\beta)\left(r-c_{p}\right)+c_{p}$.

2) Skip if Good: Transmits as long as the observed channel state is bad. If the observed state is good transmission is withheld for the following time instant after which it resumes again. This policy is optimal if $c_{d}<\alpha\left(r-c_{p}\right)+c_{p} \wedge c_{d} \geq(1-\beta)\left(r-c_{p}\right)+c_{p}$.

Proof: For the negatively correlated channels [44]:

$\tau^{2 k}(w)$ and $\tau^{2 k+1}(w) \rightarrow \pi_{g}$, from opposite directions, as $k \rightarrow \infty$.

If $w^{*} \in[\tau(1-\beta), 1]$ then transmission can be suspended forever. When the channel is observed in the good state $(w=$ $\left.1-\beta<w^{*}\right)$ then it is optimal to idle the channel. This results in the channel never being used since from (7), $\tau^{k}(1-\beta)>$ $w^{*}$, can never be satisfied. Therefore, to guarantee reliability, we should have $w^{*} \in[0, \tau(1-\beta)]$, which can be split into $[0,1-\beta] \cup\left[1-\beta, \tau(1-\beta)\left[\right.\right.$. If $w^{*} \in[0,1-\beta]$, by $1-\beta \leq w \leq \alpha$, $w>w^{*}$ is always satisfied and the optimal policy is to always use the channel. Now if $w^{*} \in[1-\beta, \tau(1-\beta)]$, then when $w=\alpha>w^{*}$ (i.e., the channel is observed to be bad) it is optimal to use it again. If the channel is good though, then $w=1-\beta$ which is not greater than $w^{*}$, meaning it is optimal to idle the channel. Consequently, in the following time instant, $w$ will be updated to $w=\tau(1-\beta)>w^{*}$ and the optimal action is to transmit over the channel.

Implications of Theorems 3 and 4. The established theorems indicate that an optimal reliable transmission protocol continues to transmit after a successful (failed) transmission along a positively (negatively) correlated channel. This same protocol, however, will wait for a fixed time, say $T_{\text {wait }}$, before attempting to send again after a failed (successful) transmission. Hence an optimal reliable protocol can be defined solely by the waiting time $T_{\text {wait }}$ after a successful (failed) sending attempt.

\section{IMPACT OF LOSSY FEEDBACK}

Recall that so far, and for presentation simplicity, we have considered perfect feedback. We now investigate the impact of lossy feedback on energy-optimal transmission policies by studying the system under the constant feedback, smart feedback and unreliable feedback mechanisms.

Transmitting over the link yields an immediate expected reward of:

$$
\begin{aligned}
w(1-\beta) & \mathcal{R}\left(a_{t_{i}}=1, o_{t_{i+2}}=a c k\right)+\alpha(1-w) \mathcal{R}\left(a_{t_{i}}=1, o_{t_{i+2}}=\text { nack }\right) \\
& +(w(1-\beta)+\alpha(1-w)) \mathcal{R}\left(a_{t_{i}}=1, o_{t_{i+2}}=\perp\right) \\
& =w(1-\beta)\left(r-c_{p}\right)+c_{p} .
\end{aligned}
$$

Idling however yields an immediate expected cost of $c_{d}$ (since no other cost/reward exists relative to idling the channel). Next we derive the value function relative for each of the other feedback mechanisms.

Constant Feedback. The future maximum expected total discounted reward, regardless if $S$ transmits or idles the link, will be either: (i) $\gamma V_{\gamma}(1-\beta)$ (if an ack or a nack is obtained) or (ii) $\gamma V_{\gamma}(\alpha)$ (if a $\perp$ is obtained). The former occurs with probability $\tau(w)$ while the latter occurs with probability $1-$ $\tau(w)$, resulting in:

$$
\begin{aligned}
V_{\gamma}(w ; a=1) & =w(1-\beta)\left(r-c_{p}\right)+c_{p} \\
& +\gamma\left[\tau(w) V_{\gamma}(1-\beta)+(1-\tau(w)) V_{\gamma}(\alpha)\right] \\
V_{\gamma}(w ; a=0)= & c_{d}+\gamma\left[\tau(w) V_{\gamma}(1-\beta)+(1-\tau(w)) V_{\gamma}(\alpha)\right] .
\end{aligned}
$$

The value function $V_{\gamma}(w)$ can be thus recursively written as:

$$
\begin{aligned}
& V_{\gamma}(w)=\max \left\{V_{\gamma}(w ; a=1), V_{\gamma}(w ; a=0)\right\} \\
& \quad=\max \left\{w(1-\beta)\left(r-c_{p}\right)+c_{p}+\gamma\left[\tau(w) V_{\gamma}(1-\beta)+(1-\tau(w)) V_{\gamma}(\alpha)\right],\right. \\
& \left.c_{d}+\gamma\left[\tau(w) V_{\gamma}(1-\beta)+(1-\tau(w)) V_{\gamma}(\alpha)\right]\right\} .
\end{aligned}
$$

Smart Feedback. Under this feedback mechanism, the future maximum expected total discounted reward, relative to $S$ transmitting over the the link, will be either: (i) $\gamma V_{\gamma}(1-\beta)$ (if an ack or a nack is obtained) or (ii) $\gamma V_{\gamma}(\alpha)$ (if a $\perp$ is obtained). The former occurs with probability $\tau(w)$ while the latter occurs with probability $1-\tau(w)$.

$$
\begin{aligned}
V_{\gamma}(w ; a=1) & =w(1-\beta)\left(r-c_{p}\right)+c_{p} \\
& +\gamma\left[\tau(w) V_{\gamma}(1-\beta)+(1-\tau(w)) V_{\gamma}(\alpha)\right]
\end{aligned}
$$

However, when $S$ does not use the link to send a message, $w$ will deterministically shift to $\tau^{2}(w)$ resulting in:

$$
V_{\gamma}(w ; a=0)=c_{d}+\gamma V_{\gamma}\left(\tau^{2}(w)\right) .
$$

So, $V_{\gamma}(w)$ can be written as:

$$
\begin{aligned}
V_{\gamma}(w) & =\max \left\{V_{\gamma}(w ; a=1), V_{\gamma}(w ; a=0)\right\} \\
& =\max \left\{w(1-\beta)\left(r-c_{p}\right)+c_{p}+\gamma\left[\tau(w) V_{\gamma}(1-\beta)+(1-\tau(w)) V_{\gamma}(\alpha)\right],\right. \\
& \left.c_{d}+\gamma V_{\gamma}\left(\tau^{2}\left(w_{t}\right)\right)\right\} .
\end{aligned}
$$

Unreliable Feedback. Under this feedback mechanism, the future maximum expected total discounted reward, relative to $S$ transmitting over the channel, will be either: (i) $\gamma V_{\gamma}(1-\beta)$ (if an ack is obtained) or (ii) $\gamma V_{\gamma}\left(\frac{\tau(\alpha)-\alpha w(2-2 \beta-\alpha)}{1-w(1-\beta)}\right)$ (if $\perp$ is obtained). The former occurs with probability $w(1-\beta)$ while the latter occurs with probability $1-w(1-\beta)$.

$$
\begin{aligned}
V_{\gamma}(w ; a=1) & =w(1-\beta)\left(r-c_{p}\right)+c_{p}+ \\
\gamma & {\left[w(1-\beta) V_{\gamma}(1-\beta)+(1-w(1-\beta)) V_{\gamma}\left(\frac{\tau(\alpha)-\alpha w(2-2 \beta-\alpha)}{1-w(1-\beta)}\right)\right] }
\end{aligned}
$$


As in the smart feedback mechanism when $S$ idles the channel, $V_{\gamma}(w ; a=1)=c_{d}+\gamma V_{\gamma}\left(\tau^{2}(w)\right)$. So, $V_{\gamma}(w)$ can be written as:

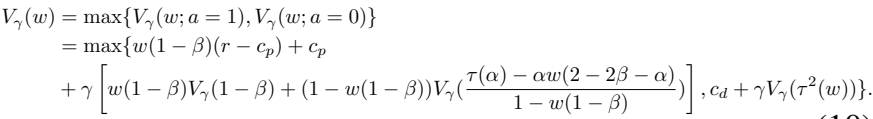

\section{Optimal Transmission Policies Under Lost Feedback}

Having obtained the value function $V_{\gamma}(w)$, we investigate the structure of the reliable energy-optimal transmission policy under the different feedback mechanisms.

Constant Feedback. By observing (8), we can note that the maximum future total expected discounted reward for using the channel and idling it is the same (denote it by $F$ ). Thus:

$$
\begin{aligned}
V_{\gamma}(w) & =\max \left\{V_{\gamma}(w ; a=1), V_{\gamma}(w ; a=0)\right\} \\
& =\max \left\{w(1-\beta)\left(r-c_{p}\right)+c_{p}+F, c_{d}+F\right\} .
\end{aligned}
$$

Theorem 5: A myopic (greedy) threshold policy is the energy-optimal transmission policy that guarantees reliable transmission under a constant feedback mechanism when $(1-\beta)^{2}>\frac{c_{d}-c_{p}}{r-c_{p}}$.

Proof: From (11), it is clear that the value function depends only on the immediate expected reward. The optimal policy can be stated as:

$$
\text { optimal policy }= \begin{cases}\text { transmit } & \text { if } w>\frac{c_{d}-c_{p}}{(1-\beta)\left(r-c_{p}\right)}, \\ \text { idle } & \text { otherwise. }\end{cases}
$$

By the update belief function under constant feedback (Section III), the optimal policy will never suspend transmission forever if $(1-\beta)^{2}>\frac{c_{d}-c_{p}}{r-c_{p}}$.

Smart Feedback. As in perfect feedback (Section V), $V_{\gamma}(w)$ for smart feedback can be shown to be a convex function in $w$ continuous on $[0,1]$.

Lemma 6: If $c_{d}<w(1-\beta)\left(r-c_{p}\right)+c_{p}$ (in particular if $c_{d}<c_{p}$ ), then the optimal decision is to use the channel for transmission at every $t$.

Proof: By convexity of $V_{\gamma}(w)$ in $w$ we have

$V_{\gamma}\left(\tau^{2}(w)\right)=V_{\gamma}(\tau(w)(1-\beta)+\alpha(1-\tau(w))) \leq \tau(w) V_{\gamma}(1-\beta)+(1-\tau(w)) V_{\gamma}(\alpha)$

$V_{\gamma}(w ; a=1)$ will be greater than $V_{\gamma}(w ; a=0)$, if $c_{d}<$ $w(1-\beta)\left(r-c_{p}\right)+c_{p}$, i.e., if $c_{d}-c_{p}<w(1-\beta)\left(r-c_{p}\right)$. Given that $w(1-\beta)\left(r-c_{p}\right) \geq 0, V_{\gamma}(w ; a=1)>V_{\gamma}(w ; a=0)$ if $c_{d}-c_{p}<0$.

Theorem 6: The energy optimal transmission policy under a smart feedback mechanism is a threshold policy, i.e., $V_{\gamma}(w ; a=1)<V_{\gamma}(w ; a=0) \forall w<w^{*}$ and $V_{\gamma}(w ; a=1)>$ $V_{\gamma}(w ; a=0) \forall w>w^{*}$ for a unique $w^{*}$, only if $c_{d}-c_{p} \geq$ $\gamma\left[\tau(\alpha) V_{\gamma}(1-\beta)+(1-\tau(\alpha)) V_{\gamma}(\alpha)-V_{\gamma}(\tau(\alpha))\right] \wedge r>\frac{c_{d}-\beta c_{p}}{1-\beta}$.

Proof: At $w=0$ :

$V_{\gamma}(0 ; a=1)=c_{p}+\gamma\left[\tau(\alpha) V_{\gamma}(1-\beta)+(1-\tau(\alpha)) V_{\gamma}(\alpha)\right]$ and $V_{\gamma}(0 ; a=0)=c_{d}+\gamma V_{\gamma}(\tau(\alpha))$. From Section III and Lemma $1 c_{d}>c_{p}$ (since $c_{d}<c_{p}$ trivializes the optimal policy to that which constantly transmits over the channel). By convexity of $V_{\gamma}(w)$ in $w, V_{\gamma}(\tau(\alpha)) \leq \tau(\alpha) V_{\gamma}(1-\beta)+$ $(1-\tau(\alpha)) V_{\gamma}(\alpha)$. Thus, if $c_{d}-c_{p}<\gamma\left[\tau(\alpha) V_{\gamma}(1-\beta)+(1-\right.$ $\left.\tau(\alpha)) V_{\gamma}(\alpha)-V_{\gamma}(\tau(\alpha))\right]$, then $V_{\gamma}(0 ; a=1)<V_{\gamma}(0 ; a=0)$; otherwise $V_{\gamma}(0 ; a=1)>V_{\gamma}(0 ; a=0)$.

At $w=1$ :

$V_{\gamma}(1 ; a=1)=r(1-\beta)+\beta c_{p}+\gamma V_{\gamma}(1-\beta)$ and $V_{\gamma}(1 ; a=$ $0)=c_{d}+\gamma V_{\gamma}(1-\beta)$. Consequently, if $r>\frac{c_{d}-\beta c_{p}}{1-\beta}$, then $V_{\gamma}(1 ; a=1)>V_{\gamma}(1 ; a=0)$; otherwise $V_{\gamma}(1 ; a=1)<$ $V_{\gamma}(1 ; a=0)$. From (9), it can be seen that $V_{\gamma}(w ; a=1)$ is linear in $w$. Following from the convexity of $V_{\gamma}(w)$, we can conclude that $V_{\gamma}(w ; a=0)$ is convex in $w$. This leads to a graph as that shown in Figure 2 concluding the proof.

Closed form solutions and conditions relative to attaining reliability can be obtained similar to those of perfect feedback Section VI. Due to space limitation we do not elaborate further on these conditions since an analogous analysis has been already conducted in details (Section VI).

Unreliable Feedback. We analyse unreliable feedback for positively correlated channels only.

Lemma 7: Under the unreliable feedback mechanism the channel belief is $w \in[0,1]$.

Proof: $w$ at any time can take one of the following values: (i) $1-\beta$, (ii) $\mathrm{T}(w)$ or (iii) $\tau(\tau(w))$. [44] shows that $\tau^{n}(w) \forall w$, tends to $\frac{\alpha}{\alpha+\beta}$ as $n \longrightarrow \infty\left(\tau^{n}(w)\right.$ means that the function $\tau$ is called $n$ times on $w$ ). It can be easily shown that $\mathrm{T}(w)$ is a decreasing function in $w$ such that $0<\mathrm{T}(1), \mathrm{T}(0)<1$.

Lemma 8: $\mathrm{T}(w)$ is a convex function in $w$.

Proof: For $0<w_{1}, w_{2}, \lambda<1$ :

$$
\begin{aligned}
\lambda \mathrm{T}\left(w_{1}\right) & +(1-\lambda) \mathrm{T}\left(w_{2}\right)-\mathrm{T}\left(\lambda w_{1}+(1-\lambda) w_{2}\right)= \\
& =(1-\beta)^{2} \tau(\alpha)\left[\lambda(1-\lambda)\left(w_{2}-w_{1}\right)^{2}\right] \\
& +(1-\beta)(2-2 \beta-\alpha)\left[\lambda w_{1}\left(1+w_{1}\left(2 w_{2}(1-\beta)-1\right)\right)\right. \\
& \left.+(1-\lambda) w_{2}\left(1+w_{2}\left(2 w_{1}(1-\beta)-1\right)\right)\right] \geq 0
\end{aligned}
$$

The value function $V_{\gamma}(w)$ can be obtained by value iteration as a uniform limit of cost functions for finite horizon problems, which are continuous and convex [42], [56]. $V_{\gamma}(w)$, as the upper envelope of a family of straight lines (cost functions), is thus convex in $w$ (proved by [57]).

Theorem 7: For a channel with a defined cost function under unreliable feedback, there will exist at least one value $w^{*}$ such that $V_{\gamma}\left(w^{*} ; a=1\right)=V_{\gamma}\left(w^{*} ; a=0\right)$ only if $r>\frac{c d-\beta c_{p}}{1-\beta}$.

Proof: At $w=0$ :

$V_{\gamma}(0 ; a=1)=c_{p}+\gamma V_{\gamma}(\tau(\alpha))$ and $V_{\gamma}(0 ; a=0)=c_{d}+$ $\gamma V_{\gamma}(\tau(\alpha))$. Since $c_{d}>c_{p}$, then $V_{\gamma}(0 ; a=1)<V_{\gamma}(0 ; a=0)$ (recall from Section III that if $c_{d}<c_{p}$ the optimal action is to constantly transmit over the channel).

At $w=1$ :

$V_{\gamma}(1 ; a=1)=r(1-\beta)+\beta c_{p}+\gamma\left[(1-\beta) V_{\gamma}(1-\beta)+\beta V_{\gamma}(\alpha)\right.$ and $V_{\gamma}(1 ; a=0)=c_{d}+\gamma V_{\gamma}(\tau(1-\beta))$. By convexity of $V_{\gamma}(w)$ we have $V_{\gamma}(\tau(1-\beta)) \leq(1-\beta) V_{\gamma}(1-\beta)+\beta V_{\gamma}(\alpha)$. If $r>\frac{c d-\beta c_{p}}{1-\beta}$ we get $V_{\gamma}(1 ; a=1)>V_{\gamma}(1 ; a=0)$.

Both $V_{\gamma}(w ; a=0)$ and $V_{\gamma}(w ; a=1)$ depend on $V_{\gamma}(f(w))$ (where $f(w)$ is some function of $w$ ) and are convex (due to the convexity of $\left.V_{\gamma}(w) \forall w\right) . V_{\gamma}(w ; a=0)$ and $V_{\gamma}(w ; a=1)$ will 

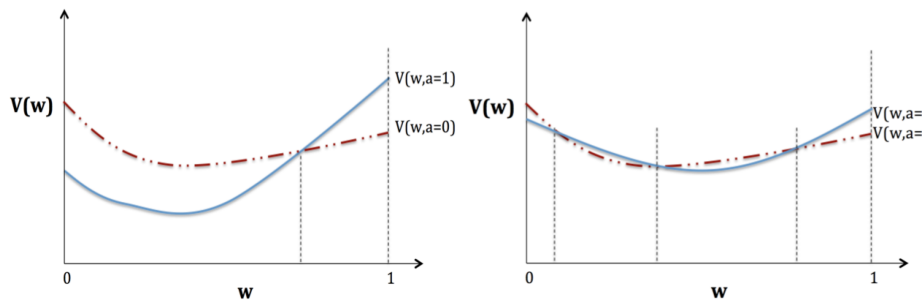

Fig. 4. Behavior of value functions for transmitting and idling under unreliable feedback.

thus intersect in at least a single point as shown in Figure 4 concluding the proof.

The optimal policy under unreliable feedback is to transmit only in the intervals where $V_{\gamma}(w ; a=0)<V_{\gamma}(w ; a=1)$, which by Figure 4 may span multiple disjoint intervals over $w \in[0,1]$. Consequently, a simple threshold policy (as that under the other feedback mechanisms) is not necessarily guaranteed, i.e., the optimal policy may not be a simple threshold policy.

\section{ESTABLISHING SYNCHRONOUS COMMUNICATION}

The transmission on a channel is subject at any time to a non-zero probability of message loss, which may lead to a finite but unbounded delivery time for messages. In this section, we show how synchronous communication over such a channel can be guaranteed with high probability. For presentation simplicity and space limitations we carry our analysis for the optimal transmission under the perfect feedback mechanism. Similar analyses can be easily conducted for the other feedback schemes. We determine the probability distribution of the total time, $X$, required to deliver the last message in the finite queue of size $N$. As such being able to guarantee with high probability that such a message is delivered within a specific time, say $\delta$, implies that any message will get delivered in time $\leq \delta$.

More precisely, define the waiting time of the $i^{t h}$ message in the queue to be the time required for this message to reach the top of the queue. Let $X_{i}$ designate the time to successfully transmit the $i^{t h}$ message in the queue, given it has zero waiting time. $X_{i}$ is thus the time it takes message $i$ to get from the top of sender's queue to the receiver's side. In queuing theory, $X_{i}$ is known as the service time. Given $N$ messages in the queue, $X=X_{1}+X_{2}+\ldots+X_{N}$. We are interested in the minimum $\delta$ such that $P[X<\delta]=1-\epsilon$. We show in what follows how to determine the value of $\delta$ for the channel.

Lemma 9: $X_{i} \forall i$, in a Gilbert-Elliot channel model, are independent and identically distributed random variables, given the initial channel belief $w_{0}=1-\beta$.

Proof: The following three facts apply to every message at the top of the queue:

1. If a message reaches the top of the queue at time $t \in \mathcal{T}$, then the channel state at $[t-1, t[$ was good. A message only reaches the top of the queue if the previous message is no longer there, i.e., has been successfully transmitted.

2. Given that the channel was good at $[t-1, t[$ the probability that the channel state stays good or becomes bad is independent of the message. Thus for any message reaching the top of the queue, the probability distribution of the channel being good is the same.

3. The optimal transmission policy is deterministic and independent of the message, but dependent on the state of the channel, which by 1 and 2 , is the same for every message reaching the top of the queue.

As a result the probability distribution of successfully transmitting a message is the probability distribution of the channel being in the good state after being observed to be good some $t$ time steps ago, where $t$ by the third fact is only dependent on the policy. Due to the fact that the transmission policy does not change for a given channel, neither do the channel stochastic parameters, this probability distribution is fixed and thus independent and identical for every message. By assuming $w_{0}=1-\beta$, the very first message will have identical distribution as well.

The probability distribution of $X, f_{X}(k)=P[X=k]$, is obtained by the convolution of the distributions of $X_{i}$ 's.

$\left.f_{X}(k)=\sum_{k_{1}=1}^{k} \sum_{k_{2}=1}^{k} \ldots \sum_{k_{N-1}=1}^{k} f_{X_{1}}\left(k_{1}\right) \cdot f_{X_{2}}\left(k_{2}\right) \ldots f_{X_{N}}\left(k-\sum_{i=1}^{N-1} k_{i}\right)\right)$.

The minimum $\delta$ such that $P[X<\delta]=1-\epsilon$ can be found by $\underset{\delta}{\operatorname{argmin}}\left\{\delta: \sum_{k=1}^{\delta} f_{X}(k)>=1-\epsilon\right\} . f_{X}(k)$ for a general queue of size $N$ is hard to express in a closed form. $f_{X}(k)$ can however be obtained offline by a simple algorithm implementing the function in (12). For theoretical interest, we alternatively obtain a closed form of an upper bound on $\delta$.

Theorem 8: The time to deliver all $N$ messages in the queue with probability $1-\epsilon$ is upper bounded by

$$
\delta=\left\lceil\frac{N \cdot E\left[X_{i}\right]}{\epsilon}\right\rceil \text {. }
$$

Proof: The average waiting time of the $N^{\text {th }}$ message is $E[X]=E\left[\sum_{i=1}^{N} X_{i}\right]=\sum_{i=1}^{N} E\left[X_{i}\right]=N \cdot E\left[X_{i}\right]$. By Markov's inequality we have $P[X>\delta]<\frac{E[X]}{\delta}=\frac{N \cdot E\left[X_{i}\right]}{\delta}$. Multiplying by -1 and then adding 1 to both sides of the inequality leads to $P[X \leq \delta]>1-\frac{N \cdot E\left[X_{i}\right]}{\delta}$. Since we need $P[X<\delta]=1-\epsilon$, then an upper bound on $\delta$ is $\underset{\delta}{\operatorname{argmin}}\left\{\delta: 1-\frac{N \cdot E\left[X_{i}\right]}{\delta} \geq 1-\epsilon\right\}$, which after simple calculation leads to $\delta=\left\lceil\frac{N \cdot E\left[X_{i}\right]}{\epsilon}\right\rceil$.

Next we compute closed form expressions of $f_{X_{i}}(k)$ and the average service time $E\left[X_{i}\right]$ for all optimal reliable policies.

Constantly transmit. A message reaches the top queue only if the message proceeding it gets successfully transmitted, inferring that the channel state was good. Since this policy always transmits, a message arriving to the top of the queue at time $t$ gets successfully transmitted at $t+1$ with probability $1-\beta$ (i.e., channel is good at $t+1$ ), at $t+2$ with probability $\beta \alpha$ (i.e., channel is bad at $t+1$ and good at $t+2$ ), so on and so forth. The probability distribution of $X_{i}$ is:

$f_{X_{i}}(k)=P\left[X_{i}=k\right]= \begin{cases}1-\beta & \text { if } k=1 \\ \beta \alpha(1-\alpha)^{k-2} & \text { if } k \in\{2,3, \ldots, \infty\} .\end{cases}$ 
The average time to successfully transmit a message on the channel given that it is on the top of the queue, $E\left[X_{i}\right]$, is:

$E\left[X_{i}\right]=\sum_{k=1}^{\infty} k \cdot f_{X_{i}}(k)=1-\beta+\sum_{t=2}^{\infty} \beta \alpha t(1-\alpha)^{t-2}=\frac{\alpha+\beta}{\alpha}$.

We illustrate for this constantly transmit policy how to compute the average number of message waiting in the queue. Assume a geometric arrival process and denote by $\lambda$ the average arrival rate per unit time. Let $\bar{\lambda}=1-\lambda$. Given a queue of fixed size $N$, we create a finite state machine (FSM) for the number of messages in the queue. The designed FSM results in states $\left\{0,1,1^{\prime}, 2,2^{\prime}, \ldots, N, N^{\prime}\right\}$, where $S$ and $S^{\prime}$ are states with the same number of messages in the queue but which may shift to different states and with different probabilities. This results from time varying behavior of the channel, which leads to service rates that change with time. We determine the transitions between these states and represent in the following transition matrix:

$$
I=\left(\begin{array}{cccccccc}
\bar{\lambda} & \lambda & 0 & 0 & 0 & 0 & 0 & \ldots \\
\bar{\lambda}(1-\beta) & \lambda(1-\beta) & \bar{\lambda} \beta & 0 & \lambda \beta & 0 & 0 & \ldots \\
\bar{\lambda} \alpha & \lambda \alpha & \bar{\lambda}(1-\alpha) & 0 & \lambda(1-\alpha) & 0 & 0 & \ldots \\
0 & \bar{\lambda}(1-\beta) & 0 & \lambda(1-\beta) & \bar{\lambda} \beta & 0 & \lambda \beta & \ldots \\
0 & \bar{\lambda} \alpha & 0 & \lambda \alpha & \bar{\lambda}(1-\alpha) & 0 & \lambda(1-\alpha) & \ldots \\
0 & 0 & 0 & \bar{\lambda}(1-\beta) & 0 & \lambda(1-\beta) & \bar{\lambda} \beta & \ldots \\
0 & 0 & 0 & \bar{\lambda} \alpha & 0 & \lambda \alpha & \bar{\lambda}(1-\alpha) & \ldots \\
\ldots & \ldots & \ldots & \ldots & \ldots & \ldots & \ldots & \ldots
\end{array}\right)
$$

The rows of the matrix correspond to states at time $t$ while the columns correspond to states at time $t+1$. Extracting the information from the transition matrix above results in the following system of equations:

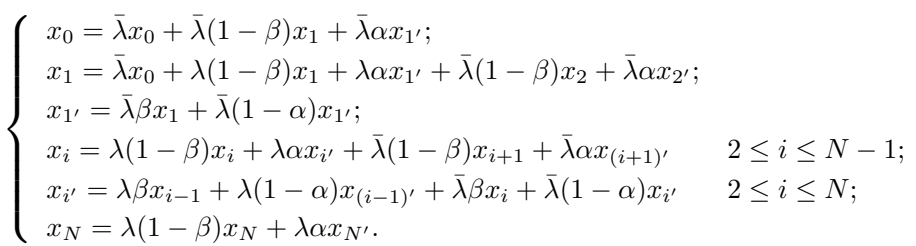

Solving this system of equations allows to determine an important value/metric, the probability of having $i$ messages in the queue: $P_{i}=x_{i}+x_{i^{\prime}}$. The average number of waiting messages in the queue can be calculated by: $\sum_{i=0}^{N} i P_{i}$.

Back-off on bad. This transmission scheme keeps transmitting on the channel as long as the observed state is good. It however ceases transmission for some time $T$ (Theorem 3) after observing the channel in the bad state after which transmission is resumed. Such form of transmission can be optimal only in positively correlated channels. The probability distribution of $X_{i}$ is:

$f_{X_{i}}(k)=P\left[X_{i}=k\right]= \begin{cases}1-\beta & \text { if } k=1, \\ \beta \tau^{\top}(\alpha)\left(1-\tau^{\top}(\alpha)\right)^{\left(\frac{k-1}{\mathrm{~T}+1}-1\right)} & \text { if } k \in\{\mathrm{T}+2,2(\mathrm{~T}+1)+1, \ldots,\end{cases}$

The average service time $E\left[X_{i}\right]$ is:

$$
\begin{aligned}
E\left[X_{i}\right] & =\sum_{k=1}^{\infty} k \cdot f_{X_{i}}(k)=(1-\beta)+\sum_{t=0}^{\infty}[(t+1) \top+1] \beta \tau^{\top}(\alpha) t\left(1-\tau^{\top}(\alpha)\right)^{t} \\
& =\frac{\beta(\top+1)+\tau^{\top}(\alpha)}{\tau^{\top}(\alpha)} .
\end{aligned}
$$

Skip if good. This policy occurs in negatively correlated channels and results in the following distribution:

$f_{X_{i}}(k)=P\left[X_{i}=k\right]= \begin{cases}\tau(1-\beta) & \text { if } k=2, \\ (1-\tau(1-\beta)) \alpha(1-\alpha)^{k-3} & \text { if } k \in\{3, \ldots, \infty\}\end{cases}$
The average service time $E\left[X_{i}\right]$ is:

$$
\begin{aligned}
E\left[X_{i}\right] & =\sum_{k=1}^{\infty} k \cdot f_{X_{i}}(k)=2 \tau(1-\beta)+\sum_{t=0}^{\infty}(t+3)(1-\tau(1-\beta)) \alpha(1-\alpha)^{t} \\
& =\frac{4(1-\tau(1-\beta))-\alpha(1-2 \tau(1-\beta))}{\alpha} .
\end{aligned}
$$

\section{CONCLUSION}

We presented in this paper an analytic study describing how energy-optimal reliable communication can be built, with high probability synchrony, over unreliable wireless channels. The analysis was conducted for a time-varying lossy channel that captures the unreliable behavior of wireless. We discussed four forms of Ack/Nack feedback mechanisms. We obtained under reliable feedback, a closed form of the policy which determines when it is optimal to transmit over the channel. Optimality in this context was achieved with respect to a defined energy-through-latency trade-off. Combined with this closed form solution, we also identified the necessary conditions under which transmission is never suspended and provided a probabilistic bound on the total time to deliver a message. Hence, we presented an implementable form of an optimal reliable communication, guaranteeing high probability synchrony. We also studied the impact of lossy feedback on optimal transmission, which to the best of our knowledge is a first. We showed that easy implementable forms of the desired communication service can also be obtained depending on the utilized feedback mechanism. Possible future work may look into a more complex channel structure, particularly, when the states of the channel are not fully reliable/unreliable, or into a case where the same channel is considered for both uplink and down-link communication, i.e. down-link not only for feedback.

\section{ACKNOWLEDGMENT}

This work was partially supported by the EU ERC project "Adversary Oriented Computing".

\section{REFERENCES}

[1] A. Czumaj, R. Elssser, L. Gsieniec, T. Sauerwald, and X. Wang, "Fast message dissemination in random geometric networks," Distributed Computing, vol. 26, no. 1, 2013.

[2] E. Latronico and P. Koopman, "Design time reliability analysis of distributed fault tolerance algorithms," in DSN, 2005.

[3] G. Veronese, M. Correia, A. Bessani, L. C. Lung, and P. Verissimo, "Efficient byzantine fault-tolerance," IEEE Trans. Comput., 2013.

[4] F. Greve and S. Tixeuil, "Conditions for the solvability of fault-tolerant consensus in asynchronous unknown networks," in WRAS, 2010.

[5] J. Aspnes, F. E. Fich, and E. Ruppert, "Relationships between broadcast and shared memory in reliable anonymous distributed systems," Distributed Computing, vol. 18, no. 3, 2006.

[6] S. Chen, K. Joshi, M. Hiltunen, R. Schlichting, and W. Sanders, "Using link gradients to predict the impact of network latency on multitier applications," IEEE/ACM Trans. on Networking, vol. 19, no. 3, 2011.

[7] C. Fetzer, "Perfect failure detection in timed asynchronous systems," IEEE Trans. Comput, vol. 52, no. 2, 2003.

[8] R. Yared, X. Défago, J. Iguchi-Cartigny, and M. Wiesmann, "Collision prevention platform for a dynamic group of asynchronous cooperative mobile robots," JNW, vol. 2, 2007.

[9] R. Rao, S. Akella, and G. Guley, "Power line carrier (plc) signal analysis of smart meters for outlier detection," in SmartGridComm, 2011.

[10] J.-P. Vasseur and A. Dunkels, Interconnecting smart objects with ip: The next internet. Morgan Kaufmann, 2010. 
[11] N. Baccour, A. Koubaa, C. Noda, H. Fotouhi, M. Alves, H. Youssef, M. Zuniga, C. A. Boano, K. Römer, D. Puccinelli, T. Voigt, and L. Mottola, Radio Link Quality Estimation in Low-Power Wireless Networks, ser. Springer Briefs in Electrical and Computer Eng., 2013.

[12] D. Dzung, R. Guerraoui, D. Kozhaya, and Y. A. Pignolet, "Source routing in time-varing lossy networks," in NETYS, 2015, pp. 200-215.

[13] O. Hohlfeld, R. Geib, and G. Hasslinger, "Packet loss in real-time services: Markovian models generating qoe impairments," in IWQoS, June 2008.

[14] G. Hasslinger and O. Hohlfeld, "The gilbert-elliott model for packet loss in real time services on the internet," in $M M B$, March 2008.

[15] Q. Han, Y. Zhang, X. Chen, H. Li, and J. Quan, "Efficient and robust identity-based handoff authentication for eap-based wireless networks," Concurrency and Computation: Prac. and Exp., vol. 26, no. 8, 2014.

[16] L. Marques and A. Casimiro, "Fighting uncertainty in highly dynamic wireless sensor networks with probabilistic models," in SRDS, 2013.

[17] T. Izumi, K. Kinpara, T. Izumi, and K. Wada, "Space-efficient selfstabilizing counting population protocols on mobile sensor networks," Theoretical Computer Science, vol. 552, 2014.

[18] A. J. Dou, S. Lin, V. Kalogeraki, and D. Gunopulos, "Supporting historic queries in sensor networks with flash storage," Information Systems, vol. 39, 2014.

[19] A. D. Kshemkalyani, A. A. Khokhar, and M. Shen, "Execution and time models for pervasive sensor networks," IJNC, vol. 2, 2012.

[20] D. Manivannan, A. Jyotiprada, and N. Sandhu, "A survey of routing protocols for wireless sensor networks," IJNGC, vol. 5, no. 2, 2014.

[21] "Link scheduling in wireless sensor networks: Distributed edge-coloring revisited," $J P D C$, vol. 68, no. 8, 2008.

[22] Y. Kwon, K. Mechitov, and G. Agha, "Design and implementation of a mobile actor platform for wireless sensor networks," in Concurrent Objects and Beyond, ser. LNCS, 2014, vol. 8665.

[23] M. Cinque, D. Cotroneo, F. Frattini, and S. Russo, "Cost-benefit analysis of virtualizing batch systems: Performance-energy-dependability tradeoffs," in UCC, 2013.

[24] E. L. Merrer, V. Gramoli, A. Kermarrec, A. C. Viana, and M. Bertier, "Energy aware self-organizing density management in wireless sensor networks," CoRR, 2008.

[25] S. Kondo, A. Kanzaki, T. Hara, and S. Nishio, "Integration of traffic reduction and sleep scheduling for energy-efficient data gathering in wireless sensor networks," Comput. Syst. Sci. Eng., vol. 28, no. 4, 2013.

[26] M. Mutschlechner, B. Li, R. Kapitza, and F. Dressler, "Using erasure codes to overcome reliability issues in energy-constrained sensor networks," in WONS, 2014.

[27] T. Szalapski and S. K. Madria, "Energy efficient distributed grouping and scaling for real-time data compression in sensor networks," in IPCCC, 2014.

[28] Y. V. Pant, T. X. Nghiem, and R. Mangharam, "Peak power reduction in hybrid energy systems with limited load forecasts," in ACC, 2014.

[29] T. Wong, T. Tsuchiya, and T. Kikuno, "An energy-efficient broadcast scheme for multihop wireless ad hoc networks using variable-range transmission power," IEICE, vol. 90-D, no. 3, 2007.

[30] I. Urteaga, N. Yu, N. Hubbell, and Q. Han, "Aware: Activity aware maintenance of communication structures for wireless sensor networks," Pervasive and Mobile Computing, vol. 13, 2014.

[31] N. Nayyar, Y. Gai, and B. Krishnamachari, "On a restless multi-armed bandit problem with non-identical arms," Allerton, 2011.

[32] E. O. Elliott, "Estimates of error rates for codes on burst-noise channels," Bell Syst. Tech. J, vol. 42, no. 5, 1963.

[33] H. S. Wang and N. Moayeri, "Finite-state markov channel-a useful model for radio communication channels," IEEE Trans. Veh. Technol., vol. 44, 1995.

[34] Q. Zhang and S. Kassam, "Finite-state markov model for rayleigh fading channels," IEEE Trans. Commun., vol. 47, no. 11, 1999.

[35] L. Kanal and A. R. K. Sastry, "Models for channels with memory and their applications to error control," Proc. IEEE, vol. 66, no. 7, 1978.

[36] H.-S. Wang and N. Moayeri, "Finite-state markov channel-a useful model for radio communication channels," IEEE Trans. Veh. Technol., vol. 44, no. 1, 1995.
[37] D. Zhang and K. Wasserman, "Transmission schemes for time-varying wireless channels with partial state observations," in INFOCOM, 2002.

[38] A. Laourine and L. Tong, "Betting on gilbert-elliot channels," IEEE Trans. Wireless Commun., vol. 9, no. 2, February 2010.

[39] C. Tang and P. K. McKinley, "Modeling multicast packet losses in wireless lans," in MSWIM, 2003.

[40] J. pierre Ebert, A. Willig, D. ing Adam Wolisz, and T. Berlin, "A gilbert-elliot bit error model and the efficient use in packet level simulation," Tech. Rep., 1999. [Online]. Available: http: //www2.tkn.tu-berlin.de/publications/papers/tkn_report02.pdf

[41] D. Dzung and Y.-A. Pignolet, "Dynamic selection of wireless/powerline links using markov decision processes," SmartGridComm, 2013.

[42] E. J. Sondik, "The optimal control of partially observable markov processes over the infinite horizon: Discounted costs," Math. Oper. Res., vol. 26 , no. 2,1978

[43] S. Guha, K. Munagala, and P. Shi, "Approximation algorithms for restless bandit problems," J. ACM, vol. 58, no. 1, 2010.

[44] K. Liu and Q. Zhao, "Indexability of restless bandit problems and optimality of whittle index for dynamic multichannel access," IEEE Trans. Inf. Theor., vol. 56, no. 11, 2010.

[45] Z. Gao, K. Nahrstedt, W. Xiang, H. Wang, and Y. Li, "Random network coding based schemes for perfect wireless packet retransmission problems in multiple channel networks," WIRELESS PERS COMMUN, vol. 69 , no. 4,2013

[46] C. H. Papadimitriou and J. N. Tsitsiklis, "The complexity of optimal queuing network control," Math. Oper. Res., vol. 24, no. 2, 1999.

[47] Q. Zhao, B. Krishnamachari, and K. Liu, "On myopic sensing for multichannel opportunistic access: structure, optimality, and performance," IEEE Trans. Wireless Commun., vol. 7, no. 12, December 2008.

[48] P. Whittle, "Restless bandits: Activity allocation in a changing world," Journal of Applied Probability, vol. 25, 1988.

[49] M. K. Aguilera, W. Chen, and S. Toueg, "Heartbeat: A timeout-free failure detector for quiescent reliable communication," Tech. Rep., 1997. [Online]. Available: http://research.microsoft.com/en-us/people/ weic/wdag97_hb.pdf

[50] R. Guerraoui, R. Olivera, and A. Schiper, "Stubborn communication channels," IC, EPFL, Tech. Rep., 1996.

[51] H. Moniz, N. Neves, M. Correia, and P. Verssimo, "Randomization can be a healer: Consensus with dynamic omission failures," in Distributed Computing, ser. LNCS, 2009, vol. 5805.

[52] H. Moniz, N. Neves, and M. Correia, "Turquois: Byzantine consensus in wireless ad hoc networks," in DSN, June 2010.

[53] L. Johnston and V. Krishnamurthy, "Opportunistic file transfer over a fading channel: A pomdp search theory formulation with optimal threshold policies," IEEE Trans. Wireless Commun., vol. 5, 2006.

[54] G. Hasslinger and O. Hohlfeld, "The gilbert-elliott model for packet loss in real time services on the internet," in $M M B$, March 2008.

[55] A. Caracas, C. Lombriser, Y. A. Pignolet, T. Kramp, T. Eirich, R. Adelsberger, and U. Hunkeler, "Energy-efficiency through micro-managing communication and optimizing sleep," in SECON, 2011.

[56] J. Ny, M. Dahleh, and E. Feron, "Multi-uav dynamic routing with partial observations using restless bandit allocation indices," P AMER CONTR CONF, 2008.

[57] S. M. Ross, Introduction to Stochastic Dynamic Programming: Probability and Mathematical. Orlando, USA: Academic Press, 1983.

\section{APPENDIX A \\ COMPUTING $w^{*}$ IN Closed Form}

For positively correlated channels:

$$
\begin{gathered}
\tau^{k}(w) \text { monotonically tends to } \pi_{g}, \text { as } k \rightarrow \infty \\
1-\beta>\pi_{g}>\alpha .
\end{gathered}
$$

Case 1: $w^{*} \geq \pi_{g}$

If $w<w^{*}$, from (14) $\tau^{k}(w) \leq w^{*}$ and thus the optimal decision is to also idle the channel. Following from (15) 
$V(\alpha)=c_{d}+\gamma c_{d}+\gamma^{2} c_{d}+\ldots=\frac{c_{d}}{1-\gamma}$. If $w>w^{*}$, then $V(w)=w\left(r-c_{p}\right)+c_{p}+\gamma[w V(1-\beta)+(1-w) V(\alpha)]$. If $\left(w^{*} \geq 1-\beta\right)$, then $V(1-\beta)=\frac{c_{d}}{1-\gamma}$. By continuity of $V(w)$ at $w^{*}$ we have

$w^{*}\left(r-c_{p}\right)+c_{p}+\gamma\left[w^{*} V(1-\beta)+\left(1-w^{*}\right) V(\alpha)\right]=c_{d}+\gamma V\left(\tau\left(w^{*}\right)\right)$, which yields: $w^{*}=\frac{c_{d}-c_{p}}{r-c_{p}}$. However, if $w^{*}<1-\beta$, then

$$
\begin{aligned}
V(1-\beta) & =(1-\beta)\left(r-c_{p}\right)+c_{p}+\gamma\left[(1-\beta) V(1-\beta)+(\beta) \frac{c_{d}}{1-\gamma}\right] \\
& =\frac{(1-\beta)\left(r-c_{p}\right)+c_{p}+\gamma \beta \frac{c_{d}}{1-\gamma}}{1-\gamma(1-\beta)} .
\end{aligned}
$$

By continuity of $V$ at $w^{*}$

$$
\begin{aligned}
w^{*}\left(r-c_{p}\right)+c_{p} & +\gamma\left[w^{*} \frac{(1-\beta)\left(r-c_{p}\right)+c_{p}+\gamma \beta \frac{c_{d}}{1-\gamma}}{1-\gamma(1-\beta)}\right. \\
& \left.+\left(1-w^{*}\right) \frac{c_{d}}{1-\gamma}\right]=\frac{c_{d}}{1-\gamma} .
\end{aligned}
$$

After reduction:

$$
\begin{gathered}
w^{*}=\frac{\left(c_{d}-c_{p}\right)(1-\gamma(1-\beta))}{r-c_{p}(1-\gamma)-\gamma c_{d}}, \\
\frac{d w^{*}}{d c_{d}}=\frac{(1-\gamma(1-\beta))\left(r-(1-\gamma) c_{p}\right)-\gamma c_{p}(1-\gamma(1-\beta))}{\left(r-c_{p}(1-\gamma)-\gamma c_{d}\right)^{2}}>0 .
\end{gathered}
$$

which shows that $w^{*}$ is a strictly increasing function of $c_{d}$.

Case 2: $w^{*}<\pi_{g}$

By (14), $\tau^{k}\left(w^{*}\right)>w^{*} \forall k>1$ thus

$$
\begin{aligned}
V\left(\tau\left(w^{*}\right)\right) & =\tau\left(w^{*}\right)\left(r-c_{p}\right)+c_{p}+\gamma\left[\tau\left(w^{*}\right) V(1-\beta)+\left(1-\tau\left(w^{*}\right)\right) V(\alpha)\right] \\
& =c_{p}+\gamma V(\alpha)+\tau\left(w^{*}\right)\left[\left(r-c_{p}\right)+\gamma(V(1-\beta)-V(\alpha))\right] .
\end{aligned}
$$

By $(15)$, we have $(1-\beta)>w^{*}$ and

$$
\begin{gathered}
\begin{aligned}
V(1-\beta) & =(1-\beta)\left(r-c_{p}\right)+c_{p}+\gamma[(1-\beta) V(1-\beta)+\beta V(\alpha)] \\
& =\frac{(1-\beta)\left(r-c_{p}\right)+c_{p}+\gamma \beta V(\alpha)}{1-\gamma(1-\beta)} .
\end{aligned} \\
\left(r-c_{p}\right)+\gamma[V(1-\beta)-V(\alpha)]=\frac{\left(r-c_{p}\right)+\gamma c_{p}-\gamma(1-\gamma) V(\alpha)}{1-\gamma(1-\beta)} .
\end{gathered}
$$

By continuity of $V$ at $w^{*}$

$$
\begin{aligned}
& c_{p}+\gamma V(\alpha)+w^{*}\left[\left(r-c_{p}\right)+\gamma[V(1-\beta)-V(\alpha)]\right. \\
& \quad=c_{d}+\gamma\left[c_{p}+\gamma V(\alpha)+\tau\left(w^{*}\right)\left[\left(r-c_{p}\right)+\gamma(V(1-\beta)-V(\alpha))\right]\right] .
\end{aligned}
$$

Replace (18) in (19)

$$
\begin{aligned}
c_{p}+\gamma & V(\alpha)+w^{*}\left[\frac{\left(r-c_{p}\right)+\gamma c_{p}-\gamma(1-\gamma) V(\alpha)}{1-\gamma(1-\beta)}\right] \\
& =c_{d}+\gamma\left[c_{p}+\gamma V(\alpha)+\tau\left(w^{*}\right)\left[\frac{\left(r-c_{p}\right)+\gamma c_{p}-\gamma(1-\gamma) V(\alpha)}{1-\gamma(1-\beta)}\right] .\right.
\end{aligned}
$$

By $\tau\left(w^{*}\right)=\alpha+w^{*}(1-\beta-\alpha)$ reduce (20) and find $w^{*}$

$$
w^{*}=1-\frac{[1-\gamma(1-\beta)]\left[r-c_{d}\right]}{[1-\gamma(1-\beta-\alpha)]\left[\left(r-c_{p}\right)+\gamma c_{p}-\gamma(1-\gamma) V(\alpha)\right]} .
$$

Thus to find the value of $w^{*}$, we have to find $V(\alpha)$. If $\left(w^{*} \leq \alpha\right)$, then

$$
\begin{aligned}
V(\alpha) & =\alpha\left(r-c_{p}\right)+c_{p}+\gamma[\alpha V(1-\beta)+(1-\alpha) V(\alpha)] \\
& =\frac{\alpha\left(r-c_{p}\right)+c_{p}+\gamma \alpha V(1-\beta)}{1-\gamma(1-\alpha)}
\end{aligned}
$$

By the assumption of positive memory, $1-\beta>\alpha$, then

$$
V(1-\beta)=\frac{(1-\beta)\left(r-c_{p}\right)+c_{p}+\gamma \beta V(\alpha)}{1-\gamma(1-\beta)} .
$$

Solving the two equations yields

$$
V(\alpha)=\frac{\alpha\left(r-c_{p}\right)+c_{p}-\gamma c_{p}(1-\beta-\alpha)}{(1-\gamma)(1-\gamma(1-\beta-\alpha))} .
$$

Replace (22) in (21), to get $w^{*}=\frac{c_{d}-c_{p}}{r-c_{p}}$. It is easy to see that $w^{*}$ is strictly increasing function in $c_{d}$.

If $\left(w^{*}>\alpha\right)$, then by (14), for some $k$ we have

$$
\tau^{k}(\alpha)<w^{*} \leq \tau^{k+1}(\alpha) .
$$

Thus,

$$
\begin{aligned}
V(\alpha) & =c_{d}+\gamma c_{d}+\ldots+\gamma^{k} c_{d}+\alpha^{k+1} V\left(\tau^{k+1}(\alpha)\right) \\
& =\frac{1-\gamma^{k+1}}{1-\gamma} c_{d}+\gamma^{k+1} V\left(\tau^{k+1}(\alpha)\right) . \\
V\left(\tau^{k+1}(\alpha)\right) & =c_{p}+\gamma V(\alpha)+\tau^{k+1}(\alpha)\left[\left(r-c_{p}\right) \gamma(V(1-\beta)-V(\alpha))\right] \\
& =c_{p}+\gamma V(\alpha)+\tau^{k+1}(\alpha)\left[\frac{\left(r-c_{p}\right)+\gamma c_{p}-\gamma(1-\gamma) V(\alpha)}{1-\gamma(1-\beta)}\right] .
\end{aligned}
$$

Replace (24) in (23)

$$
\begin{aligned}
\left(r-c_{p}\right) & +\gamma c_{p}-\gamma(1-\gamma) V(\alpha)= \\
& =\frac{[1-\gamma(1-\beta)]\left[r\left(1-\gamma^{k+2}\right)-c_{p}(1-\gamma)-\gamma\left(1-\gamma^{k+1}\right) c_{d}\right]}{\left(1-\gamma^{k+2}\right)(1-\gamma(1-\beta))+\gamma^{k+2}(1-\gamma) \tau^{k+1}(\alpha)} .
\end{aligned}
$$

Replace (25) in (21)

$w^{*}=1-\frac{\left[r-c_{d}\right]\left[\left(1-\gamma^{k+2}\right)(1-\gamma(1-\beta))+\gamma^{k+2}(1-\gamma) \tau^{k+1}(\alpha)\right]}{[1-\gamma(1-\beta-\alpha)]\left[r\left(1-\gamma^{k+2}\right)-c_{p}(1-\gamma)-\gamma\left(1-\gamma^{k+1}\right) c_{d}\right]}$.

To write $w^{*}$ in a more readable form, let:

$A(k)=\frac{\left(1-\gamma^{k+2}\right)(1-\gamma(1-\beta))+\gamma^{k+2}(1-\gamma) \tau^{k+1}(\alpha)}{1-\gamma(1-\beta-\alpha)}$,

$B(k)=r\left(1-\gamma^{k+2}\right)-c_{p}(1-\gamma), D(k)=\gamma\left(1-\gamma^{k+1}\right)$.

$w^{*}$ can then be written as

$$
w^{*}=1-A(k) \frac{r-c_{d}}{B(k)-D(k) c_{d}} .
$$

We can show that $w^{*}$ is strictly increasing in $c_{d}$ by

$$
\frac{d w^{*}}{d c_{d}}=A(k) \frac{\left(r-c_{p}\right)(1-\gamma)}{\left(B(k)-D(k) c_{d}\right)^{2}}>0 .
$$

Since $\tau^{k}(\alpha)<w^{*} \leq \tau^{k+1}(\alpha)$ where

$\tau^{k}(w)=\pi_{g}-(1-\beta-\alpha)^{k}\left(\pi_{g}-w\right)$, then $k=\left\lceil\frac{\ln \left(1-\frac{w^{*}}{\pi_{g}}\right)}{\ln (1-\beta-\alpha)}\right\rceil-2$.

This concludes all possible cases when the channel has a positive memory. Similar analysis and computations are carried for the negatively correlated case.

\section{Negatively Correlated:}

When the channel has a negative memory, i.e. $1-\beta-\alpha<0$, we study the following cases:

Case1: $1-\beta=0 ; \alpha=1$

$$
\left\{\begin{array}{l}
V(1)=r+\gamma V(0) \\
V(0)=c_{d}+\gamma V(1)
\end{array}\right.
$$

We solve the two equations and obtain

$$
\left\{\begin{array}{l}
V(1)=\frac{r+\gamma c_{d}}{1-\gamma^{2}} \\
V(0)=\frac{c_{d}+\gamma r}{1-\gamma^{2}}
\end{array}\right.
$$


$\operatorname{if}\left(w^{*} \geq 0.5\right)$ This means that $1-w^{*}<w^{*}, \tau\left(w^{*}\right)=1-w^{*}$ and $\tau\left(1-w^{*}\right)=w^{*}$

$$
V\left(1-w^{*}\right)=c_{d}+\gamma V\left(w^{*}\right) .
$$

By continuity of $V$ at $w^{*}$ we have

$$
\begin{gathered}
V\left(w^{*}\right)=c_{d}+\gamma V\left(1-w^{*}\right) \\
=c_{d}+\gamma\left(c_{d}+\gamma V\left(w^{*}\right)\right) \\
=\frac{c_{d}}{1-\gamma} . \\
\frac{c_{d}}{1-\gamma}=w^{*}\left(r-c_{p}\right)+c_{p}+\gamma\left[w^{*} V(0)+\left(1-w^{*}\right) V(1)\right] \\
=c_{p}+\gamma \frac{r+\gamma c_{d}}{1-\gamma^{2}}+w^{*}\left[\left(r-c_{p}\right)+\gamma\left[\frac{c_{d}+\gamma r}{1-\gamma^{2}}-\frac{r+\gamma c_{d}}{1-\gamma^{2}}\right] .\right. \\
\frac{w^{*}=\frac{c_{d}\left(1+\gamma-\gamma^{2}\right)-\gamma r-c_{p}\left(1-\gamma^{2}\right)}{(1-\gamma)\left[\gamma c_{d}+r-c_{p}(1+\gamma)\right]} .}{(1-\gamma)^{2}\left[\gamma c_{d}+r-c_{p}(1+\gamma)\right]^{2}}=\frac{\left(1+\gamma-\gamma^{2}\right)(1-\gamma) r+\gamma^{2}(1-\gamma) r-c_{p}\left(1-\gamma^{2}\right)}{\left(1+c_{d}\right.}=\frac{0 .}{(30)}
\end{gathered}
$$

It can also be seen that $w^{*}$ is strictly increasing in $c_{d}$.

if $\left(w^{*}<0.5\right)$

This means $\left(1-w^{*}\right)>w^{*}$, so

$V\left(1-w^{*}\right)=\left(1-w^{*}\right)\left(r-c_{p}\right)+c_{p}+\gamma\left[\left(1-w^{*}\right) V(0)+w^{*} V(1)\right]$

and by the continuity of $V$ at $w^{*}$

$$
\begin{aligned}
& w^{*}\left(r-c_{p}\right)+c_{p}+\gamma\left[w^{*} V(0)+\left(1-w^{*}\right) V(1)\right] \\
& =c_{d}+\gamma V\left(1-w^{*}\right) \\
& =c_{d}+\gamma\left[\left(1-w^{*}\right)\left(r-c_{p}\right)+c_{p}+\gamma\left[\left(1-w^{*}\right) V(0)+w^{*} V(1)\right]\right] \\
& w^{*}=\frac{c_{d}-c_{p}+\gamma(r+\gamma V(0)-V(1))}{(1+\gamma)\left[r-c_{p}+\gamma(V(0)-V(1))\right]} \\
& =\frac{c_{d}-c_{p}+\gamma^{3} r}{\left(1-\gamma^{2}\right)\left[\gamma c_{d}+r-c_{p}(1+\gamma)\right]} \text {. } \\
& \frac{d w^{*}}{d c_{d}}=\frac{\left(1-\gamma^{2}\right)\left(1-\gamma^{4}\right) r-c_{p}\left(1-\gamma^{2}\right)}{\left(1-\gamma^{2}\right)^{2}\left[\gamma c_{d}+r-c_{p}(1+\gamma)\right]^{2}}>0 .
\end{aligned}
$$

which is strictly increasing in $c_{d}$.

Case2: $0<\alpha-(1-\beta)<1$

In this case for any $w \in[0,1], \tau^{2 k}(w)$ and $\tau^{2 k+1}(w)$ converge from opposite directions to $\pi_{g}$ as $k \rightarrow \infty$.

If $\left(w^{*} \geq \pi_{g}\right)$

Then $\tau^{k}\left(w^{*}\right)<w^{*}, \forall k$.

$$
V\left(\tau\left(w^{*}\right)\right)=\frac{c_{d}}{1-\gamma}
$$

By continuity of $V(w)$ at $w^{*}$ we have

$$
\begin{gathered}
w^{*}\left(r-c_{p}\right)+c_{p}+\gamma\left[w^{*} V(1-\beta)+\left(1-w^{*}\right) V(\alpha)\right]=c_{d}+\gamma V\left(\tau\left(w^{*}\right)\right) . \\
w^{*}=\frac{\frac{c_{d}}{1-\gamma}-c_{p}-\gamma V(\alpha)}{r-c_{p}+\gamma(V(1-\beta)-V(\alpha))} .
\end{gathered}
$$

Following from $0<\alpha-1-\beta<1$, we have $1-\beta<w_{0}$ and $\alpha>\pi_{g}$. So

$$
V(1-\beta)=c_{d}+\gamma V(\tau(1-\beta)) .
$$

$\operatorname{If}\left(\tau(1-\beta) \leq \pi_{g}\right) \tau^{k}(1-\beta)<w^{*}$, and hence

$$
V(\tau(1-\beta))=\frac{c_{d}}{1-\gamma}
$$

$\operatorname{If}\left(\tau(1-\beta)>\pi_{g}\right)$

$V(\tau(1-\beta))=\tau(1-\beta)\left(r-c_{p}\right)+c_{p}+\gamma[\tau(1-\beta) V(1-\beta)+(1-\tau(1-\beta)) V(\alpha)]$.

If $\left(\alpha \leq w^{*}\right)$ We have $\tau^{k}(\alpha)<w^{*}, \forall k$, and thus

$$
V(\alpha)=\frac{c_{d}}{1-\gamma}
$$

If $\left(\alpha>w^{*}\right)$

$$
\begin{gathered}
\left.V(\alpha)=\alpha\left(r-c_{p}\right)+c_{p}+\gamma[\alpha V(1-\beta)+(1-\alpha)) V(\alpha)\right] \\
=\frac{\alpha\left(r-c_{p}\right)+c_{p}+\gamma \alpha V(1-\beta)}{1-\gamma(1-\alpha)} . \\
r-c_{p}+\gamma(V(1-\beta)-V(\alpha)) \\
=\frac{\gamma(1-\gamma) V(1-\beta)+(1-\gamma) r-c_{p}}{1-\gamma(1-\alpha)} .
\end{gathered}
$$

Given that

$$
\tau(1-\beta)=\alpha-(1-\beta)(\alpha-1+\beta)<\alpha,
$$

divide the interval $\left[\pi_{g}, 1\right]$ into the 3 sub-intervals $\left[\pi_{g}, \tau(1-\beta)\right]$, $[\tau(1-\beta), \alpha]$ and $[\alpha, 1]$.

If $\left(w^{*} \in[\alpha, 1]\right)$

$V(\alpha)=V(1-\beta)=\frac{c_{d}}{1-\gamma}$ and thus by replacing in (34) we get

$$
w^{*}=\frac{c_{d}-c_{p}}{r-c_{p}} .
$$

If $\left(w^{*} \in[\tau(1-\beta), \alpha]\right)$

$V(1-\beta)=\frac{c_{d}}{1-\gamma}$ and

$$
\begin{gathered}
w^{*}=\frac{\frac{c_{d}}{1-\gamma}-c_{p}-\gamma \frac{\alpha\left(r-c_{p}\right)+c_{p}+\gamma \alpha V(1-\beta)}{1-\gamma(1-\alpha)}}{\frac{\gamma(1-\gamma) V(1-\beta)+(1-\gamma) r-c_{p}}{1-\gamma(1-\alpha)}} \\
=\frac{c_{d}(1+\alpha \gamma)-\gamma \alpha r-c_{p}}{\gamma c_{d}+(1-\gamma) r-c_{p}} . \\
\frac{d w^{*}}{d c_{d}}=\frac{(1+\alpha \gamma)(1-\gamma) r+\alpha \gamma^{2} r-c_{p}\left(1+\gamma^{2}\right)}{\left(\gamma c_{d}+(1-\gamma) r-c_{p}\right)^{2}}>0 .
\end{gathered}
$$

which is strictly increasing in $c_{d}$.

If $\left(w^{*} \in\left[\pi_{g}, \tau(1-\beta)\right]\right)$

$$
\begin{gathered}
V(\alpha)=\frac{\alpha(r+\gamma V(1-\beta))+c_{p}(1-\alpha)}{1-\gamma(1-\alpha)} \\
r-c_{p}+\gamma(V(1-\beta)-V(\alpha))=\frac{(1-\gamma)(r+\gamma V(1-\beta))-c_{p}}{1-\gamma(1-\alpha)} . \\
r+V(1-\beta)=c_{d}-\gamma c_{p}+\gamma^{2} V(\alpha)+\gamma \tau(1-\beta)\left[r-c_{p}+\gamma(V(1-\beta)-V(\alpha))\right] \\
=r+\gamma c_{d}+\frac{r+\gamma V(1-\beta)}{1-\gamma(1-\alpha)}\left(\gamma^{3} \alpha+\gamma^{2}(1-\gamma) \tau(1-\beta)\right) \\
+\gamma^{2} \frac{1-\tau(1-\beta)}{1-\gamma(1-\alpha)} c_{p} .
\end{gathered}
$$

Replace in (34)

$$
\begin{gathered}
w^{*}=\frac{\left(1+\alpha \gamma(1-\gamma)+\gamma^{2}(1-\beta)(\alpha-(1-\beta))\right) c_{d}-\gamma \alpha r}{(1-\gamma)\left(\gamma c_{d}+r-c_{p}(1+\gamma)\right)} \\
-\frac{c_{p}\left(1-\alpha \gamma^{2}+\gamma^{2}(1-\beta)(\alpha-(1-\beta))\right)}{(1-\gamma)\left(\gamma c_{d}+r-c_{p}(1+\gamma)\right)} .
\end{gathered}
$$


which can be shown to be strictly increasing in $c_{d}$.

If $\left(w^{*}<\pi_{g}\right)$

$$
\begin{gathered}
\left.V(\alpha)=\alpha\left(r-c_{p}\right)+c_{p}+\gamma[\alpha V(1-\beta)+(1-\alpha)) V(\alpha)\right] \\
=\frac{\alpha\left(r-c_{p}\right)+c_{p}+\gamma \alpha V(1-\beta)}{1-\gamma(1-\alpha)} . \\
r-c_{p}+\gamma(V(1-\beta)-V(\alpha)) \\
=\frac{\gamma(1-\gamma) V(1-\beta)+(1-\gamma) r-c_{p}}{1-\gamma(1-\alpha)} .
\end{gathered}
$$

$\tau\left(w^{*}\right)>\pi_{g}>w^{*}$ and thus by continuity of $V(w)$ at $w^{*}$, we obtain

$$
\begin{gathered}
w^{*}=\frac{c_{d}-(1-\gamma)\left(c_{p}+\gamma V(\alpha)\right)+\gamma \alpha\left[r-c_{p}+\gamma(V(1-\beta)-V(\alpha))\right]}{\left[r-c_{p}+\gamma(V(1-\beta)-V(\alpha))\right][1-\gamma(1-\beta-\alpha)]} \\
=\frac{[1-\gamma(1-\alpha)]\left[c_{d}-c_{p}\right]}{\left[\gamma(1-\gamma) V(1-\beta)+(1-\gamma) r-c_{p}\right][1-\gamma(1-\beta-\alpha)]} . \\
\operatorname{If}\left(0 \leq w^{*} \leq 1-\beta\right) \\
V(1-\beta)=\frac{(1-\beta)\left(r-c_{p}\right)+c_{p}+\beta \gamma V(\alpha)}{1-\gamma(1-\beta)} \\
=\frac{r[1-\beta-\gamma(1-\beta-\alpha)]+\beta c_{p}}{(1-\gamma)[1-\gamma(1-\alpha)(1-\beta)]} .
\end{gathered}
$$

Thus $w^{*}$ is found to be

$$
\begin{aligned}
w^{*} & =\frac{[1-\gamma(1-\alpha)]\left[c_{d}-c_{p}\right]}{[1-\gamma(1-\alpha)]\left(r-c_{p}\right)} \\
& =\frac{c_{d}-c_{p}}{\left(r-c_{p}\right)} .
\end{aligned}
$$

It can be clearly noticed that $w^{*}$ is strictly increasing in $c_{d}$. If $\left(1-\beta<w^{*}<\pi_{g}\right)$

Then $\tau(1-\beta)>w^{*}$ and we have

$r+\gamma V(1-\beta)=\frac{\left(\gamma c_{d}+r\right)[1-\gamma(1-\alpha)]+\gamma^{2}(1-\tau(1-\beta)) c_{p}}{(1-\gamma)\left[1-\alpha \gamma+\gamma^{2}(1-\beta)(\alpha-(1-\beta))\right]}$.

So the value of $w^{*}$ would be

$$
w^{*}=\frac{\left(c_{d}-c_{p}\right)(1+\gamma(1-\beta))}{\left[\gamma c_{d}-(1+\gamma) c_{p}+r\right]} .
$$

whose derivative with respect to $c_{d}$ is positive, meaning that $w^{*}$ is strictly increasing in $c_{d}$. This concludes the negative correlated case. 